ESAIM: M2AN 47 (2013) 1627-1655

DOI: $10.1051 / \mathrm{m} 2 \mathrm{an} / 2013081$
ESAIM: Mathematical Modelling and Numerical Analysis

www.esaim-m2an.org

\title{
TWO SHALLOW-WATER TYPE MODELS FOR VISCOELASTIC FLOWS FROM KINETIC THEORY FOR POLYMERS SOLUTIONS
}

\author{
Gladys Narbona-ReinA ${ }^{1}$ And Didier Bresch ${ }^{2}$
}

\begin{abstract}
In this work, depending on the relation between the Deborah, the Reynolds and the aspect ratio numbers, we formally derived shallow-water type systems starting from a micro-macro description for non-Newtonian fluids in a thin domain governed by an elastic dumbbell type model with a slip boundary condition at the bottom. The result has been announced by the authors in [G. Narbona-Reina, D. Bresch, Numer. Math. and Advanced Appl. Springer Verlag (2010)] and in the present paper, we provide a self-contained description, complete formal derivations and various numerical computations. In particular, we extend to FENE type systems the derivation of shallowwater models for Newtonian fluids that we can find for instance in [J.-F. Gerbeau, B. Perthame, Discrete Contin. Dyn. Syst. (2001)] which assume an appropriate relation between the Reynolds number and the aspect ratio with slip boundary condition at the bottom. Under a radial hypothesis at the leading order, for small Deborah number, we find an interesting formulation where polymeric effect changes the drag term in the second order shallow-water formulation (obtained by J.-F. Gerbeau, B. Perthame). We also discuss intermediate Deborah number with a fixed Reynolds number where a strong coupling is found through a nonlinear time-dependent Fokker-Planck equation. This generalizes, at a formal level, the derivation in [L. Chupin, Meth. Appl. Anal. (2009)] including non-linear effects (shallow-water framework).
\end{abstract}

Mathematics Subject Classification. 76A05, 76A10, 35Q84, 82D60, 74D10, 35Q30, 78M35.

Received November 8, 2010. Revised September 21, 2012.

Published online August 26, 2013.

\section{INTRODUCTION}

In this paper, we formally derived shallow-water type systems starting from a FENE type micro-macro description for non-Newtonian fluids in a thin domain. Depending on the relation between the Deborah number $\mathcal{D} e$, the Reynolds number Re and the aspect ratio $\varepsilon$, we obtain two asymptotic models: Case 1: Low Deborah number regime namely $\mathcal{D} e=\mathcal{O}(\varepsilon)$ with $1 / \operatorname{Re}=\mathcal{O}(\varepsilon)$ (asymptotically inviscid flows); Case 2: Intermediate Deborah number regime $\mathcal{D} e=\mathcal{O}(1)$ with $\operatorname{Re}=\mathcal{O}(1)$ (asymptotically viscous flows).

Our work is an extensive version of what has been announced in our proceeding [31]. Regarding the derivation of shallow-water type models for non-Newtonian fluids, we can mention the work performed in [6] with bottom

Keywords and phrases. Viscoelastic flows, polymers, Fokker-Planck equation, non Newtonian fluids, Deborah number, shallow-water system.

1 Departamento de Matemática Aplicada I, E.T.S. Arquitectura, Universidad de Sevilla, Avda. Reina Mercedes 2, 41012 Sevilla, Spain. gnarbona@us.es

2 LAMA, UMR5127 CNRS, Université de Savoie, 73376 Le Bourget du Lac, France. Didier.Bresch@univ-savoie.fr 
slip condition and by Fernández-Nieto et al. in [14] with no slip boundary condition at the bottom for a Bingham fluid. Remark that, in this last paper, an interesting non-intuitive term which takes into account the plasticity appears at main order in their shallow-water type system. This model has been also treated by Balmforth et al. [2] so as the numerical approximation of Herschel-Bulkley models, for example in the paper written by Ancey [1]. Some other results related to the approximation of non-Newtonian fluids are based in the resolution of the Navier-Stokes equations together with a rheological model, see for example [25,36]. We also mention the interesting recent paper by Bouchut and Boyaval, see [5], where they propose a reduced model for gravity-driven free-surface flows of shallow-water elastic fluids which is close to ours. The main difference comes from the fact that they consider a macroscopic description of non-Newtonian fluids (upper-convected Maxwell model) instead of a micro-macro description as in our case and a different asymptotic regime. The micro-macro model we take into account, involves a Navier-Stokes/Fokker-Planck coupled system which corresponds to a FENE elastic dumbbell model. We derive thin film models generalizing results by Gerbeau, Perthame, see [15] and F.Marche, see [28] which consider Newtonian fluids with slip boundary condition at the bottom and appropriate range of adimensionnalized numbers. It could be interesting to perform similar asymptotic in Vila's framework, see $[7,11]$ namely with no-slip boundary condition and infinitely large Reynolds number in the limit of infinitely thin layers. See [14] for such generalization but in the Bingham framework. These last works consider fluid flow on a slope for different range of coefficients. We also generalize to the free surface framework the paper [10] by Chupin which considers such kind of flows in a fixed thin domain with given velocity in the Fokker-Planck equation, so the hydrodynamic and the rheological part are treated separately. The reader is also referred to the interesting paper [12], by Degond, Lemou, Picasso, where they discuss about viscoelastic fluid models derived from kinetic equations for polymers. In particular, in this paper an ansatz is performed to find an asymptotic solution of the Fokker-Planck equation, which plays a crucial role in our work. Our result mix the two approaches by [12] and [15] to deduce the appropriate shallow-water equations under a radial hypothesis at the leading order. Depending on the assumptions made on the adimensionnalized numbers namely the Deborah, the Reynolds and the aspect ratio numbers (Cases 1 and 2 mentioned above), the expansions have to be done up to first order or second order with respect to $\varepsilon$ taking care of the Fokker-Planck equation. This is the originality compared to [5] and various original difficulties that occur dealing with Fokker-Planck equation.

Case 1. Under a radial assumption at the leading order, that is, the probability density function for the dumbbell configuration at the leading order is assumed to depend only on the length of the dumbbell, the classical hypothesis that horizontal velocity $v$ does not depend on the vertical variable $z$ at the leading order order occurs since we prove that the symmetric part of the stress tensor $\sigma$ vanishes at main order. This allows to perform the asymptotic analysis of the system following steps given in [15] even if the assumption $1 / \operatorname{Re}=\mathcal{O}(\varepsilon)$ is restrictive. In particular, when only first order is considered we don't obtain neither the viscous nor the polymer effects, it is necessary to look at the second order approximation to see some effect. The main originality is that polymeric effect is at same order than viscous effect changing the drag term in the shallow-water system through a new variable $n_{0}$ solution of a transport equation. Radial assumption allows to compute explicit solution through the Maxwellian.

Case 2. When Deborah and Reynolds numbers are fixed and only the aspect ratio goes to zero, we get at main order a viscous shallow-water system coupled with a Fokker-Planck type system to determine the microscopic effect. Due to the strong non-Newtonian component in this case, the system becomes a complex non-linear coupled system. If the viscosity coming from the Newtonian component of the fluid is not taken into account, as observed in [5], a similar expression for the shallow-water equation is obtained in ours. The difference relies on the definition of the stress tensor $\sigma_{P}$ given by (2.4) that is related to the forces acting over the polymers. In our case this definition is given through the Fokker-Planck equation - microscopic model- and in [5] comes from a macroscopic model, the Upper-Convected Maxwell model. Nevertheless the same structure for the final model is found. This generalizes [10] where a non-time dependent linear equation is obtained for the velocity in his range of coefficients and consequently none coupling is taking into account. 
The paper is organized as follows: In Section 2, we present the micro-macro initial model and the asymptotic systems we will obtain. Then we present the adimensionnalization in Section 3. Section 4 is devoted to the Low Deborah number regime with a radial assumption at the leading order. Without such assumption, we give comments. In Section 5, we consider intermediate Deborah number regime and explain why we get a stronger coupled system. Section 6 is devoted to numerical results namely we prove how even for the Low Deborah number regime, we can have an interesting effect comparable at the same order than the one derived in [15].

\section{The MiCRO-MACRO INITIAL MODEL AND THE ASYMPTOTIC SYSTEMS}

In this section, we present the micro-macro system and the boundary conditions on which we will work on and we give the different shallow-water type systems which will be obtained depending on the range of the coefficients.

\subsection{The micro-macro initial model}

\subsubsection{Navier-Stokes/Fokker-Planck system}

Let us consider a non-Newtonian flow with constant density $\rho$, in a two dimensional domain $\Omega_{t} \subset \mathbb{R}^{2}$ for all $t \in[0, T]$, given later on, governed by the equations:

$$
\rho\left(\partial_{t} u+\operatorname{div}(u \otimes u)\right)+\nabla p=\operatorname{div} \sigma-\rho g e_{z} \quad \text { and } \operatorname{div} u=0 \quad \text { in } \Omega_{t}
$$

where $u=(v, w)$ is the velocity field, $p$ the pressure, $g$ the constant gravity and $e_{z}$ the unitary normal vector in the vertical direction. The total stress tensor $\sigma$ is given by

$$
\sigma=\sigma_{S}+\sigma_{P}
$$

where $\sigma_{S}$ is the stress tensor corresponding to the Newtonian part

$$
\sigma_{S}=\mu\left(\nabla u+\nabla^{t} u\right),
$$

with $\mu$ the fluid's viscosity. The stress tensor $\sigma_{P}$ is directly related to the forces acting over the polymer through the following expression ( $c f .[9])$ :

$$
\sigma_{P}(t, x)=n_{p}\langle F(q) \otimes q\rangle_{d}-\kappa \theta n_{p}\langle\mathrm{Id}\rangle_{d},
$$

where $\langle\cdot\rangle_{d}$ is the $q$-average defined as $\langle\varphi\rangle_{d}=\int_{\mathcal{B}} \varphi(q) f(q) \mathrm{d} q$ for any function $\varphi$. The variable $q$ simply represents the vector connecting the two beads of the polymer: Elastic dumbbell polymer and $f(t, x, q)$ represents the probability at the time $t$ of finding a dumbbell at the point $x$ with elongation $q$. The other quantities involved in this expression are:

- $n_{p}$ : the number of polymer molecules per unit volume.

- $\theta$ : The constant temperature.

- $\kappa$ : The Boltzman constant (relationship between the temperature and the energy).

- $F(q)$ : The spring force (which will be specified later on)

- $\mathcal{B}$ : The range for the elongation $q$.

The kinetic theory gives us the behavior of these polymers into the fluid through the distribution function $f(t, x, q)$. The diffusion equation for this function is called Fokker-Planck equation and reads, for all $(t, q) \in$ $\mathbb{R}^{+} \times \mathcal{B}:$

$$
\partial_{t} f+u \cdot \nabla f=-\operatorname{div}_{q}\left(\nabla u \cdot q f-\frac{2}{\zeta} F(q) f-\frac{2 \kappa \theta}{\zeta} \nabla_{q} f\right),
$$

where $u$ is a given velocity field depending on $(t,(x, z))$ with $(x, z) \in \Omega_{t}$. The first term in the right-hand side is due to the hydrodynamic drag force, the second one modelizes the intramolecular force and the last one the Brownian force. The constant $\zeta$ represents the friction coefficient between the beads, it is related to the Deborah number. Readers interested by physical book for more details are referred to the one by Bird et al., see [9]. 


\subsubsection{Spring force expressions}

In this section we focus on the definition of the spring force $F(q)$ that depends on the structure of the polymers. We center in the particular case when an elastic dumbbell model is considered, for these polymers we can find two kind of elastic connectors, linear and nonlinear. Usually the spring force $F(q)$ is defined as the gradient of the called spring potential energy $P(q)$ :

$$
F(q)=\nabla_{q} P(q) .
$$

In this case and for a steady state, homogeneous, potential flow, a solution of the Fokker-Planck equation can be found:

$$
f(q)=f_{e q}(q) \phi(q)
$$

with $f_{e q}(q)$ the equilibrium distribution function,

$$
f_{e q}(q)=\frac{1}{J_{e q}} \mathrm{e}^{-\frac{P(q)}{\kappa \theta}}
$$

and

$$
\phi(q)=\frac{J_{e q}}{J} \mathrm{e}^{\frac{\zeta}{4 \kappa \theta}(\nabla u: q q)}
$$

a dimensionless factor that contains information about the flow pattern. In these expressions

$$
J_{e q}=\int_{\mathcal{B}} \mathrm{e}^{-\frac{P(q)}{\kappa \theta}} \mathrm{d} q
$$

is the normalization constant for the equilibrium distribution function $f_{e q}(q)$ and $J$ is that for the nonequilibrium distribute function; with $J$ depending on $P(q)$ and $\nabla u$. See [9] for details.

Hookean spring. For the linear case we have the Hookean spring connector for what the spring force is proportional to the beads separation:

$$
F(q)=\mathcal{H} q
$$

with $\mathcal{H}$ the spring constant. Note that we can write $F(q)$ as a potential spring force by simply taking $P(q)=$ $\frac{1}{2} \mathcal{H} q^{2}$. Thanks to this fact and the linearity of the spring force we can directly obtain a solution of the distribution function $f$ as above.

FENE spring. Since there is no restriction on $q$, the Hookean model permits an infinity elongation for the polymer, so it is not a realistic model. To avoid this trouble the following spring force is defined:

$$
F(q)=\frac{\mathcal{H} q}{1-\frac{q^{2}}{q_{m}^{2}}}, \quad q \leq q_{m} .
$$

In this case $q_{m}$ is the maximum elongation of the spring in the polymer so we find a nonlinear force but the elongation remains bounded. It is called the Finitely Extensible Nonlinear Elastic (FENE) connector force (see H.C. Ottinger, [34]). Some recent mathematical results about well-posedness of the FENE Fokker-Planck equation can be found in $[10,26,29,39]$. This is the model that we will take into account in the sequel.

\subsubsection{Coupled FENE type system with free boundary conditions}

To write the whole model we must define the domain $\Omega_{t}$ (free surface domain) and add to system (2.1)-(2.6) appropriate boundary conditions: Free surface condition and effect of the atmospheric pressure on the surface level given by $z=H$; no penetration condition and friction effect on the fixed bottom $z=z_{b}$. In conclusion, the unknowns $(u=(v, w), p, f, H)$ are solutions of the following system for all $t \in[0, T]$ :

$$
\begin{cases}\rho\left(\partial_{t} u+\operatorname{div}(u \otimes u)\right)+\nabla p=\operatorname{div} \sigma_{S}+\operatorname{div} \sigma_{P}-\rho g e_{z} ; & \text { in } \Omega_{t} \\ \operatorname{div} u=0 & \text { in } \Omega_{t}\end{cases}
$$




$$
\begin{aligned}
& \begin{cases}\sigma_{S}=\mu\left(\nabla u+\nabla^{t} u\right) \\
\sigma_{P}=n_{p} \int_{\mathcal{B}}\left(\frac{\mathcal{H} q}{1-\frac{q^{2}}{q_{m}^{2}}} \otimes q\right) f(q)-\kappa \theta n_{p} \int_{\mathcal{B}} f(q) \operatorname{Id} & \text { in } \Omega_{t}\end{cases} \\
& \partial_{t} f+u \cdot \nabla f=-\nabla_{q} \cdot\left(\nabla u \cdot q f-\frac{2}{\zeta} \frac{\mathcal{H} q}{1-\frac{q^{2}}{q_{m}^{2}}} f-\frac{2 \kappa \theta}{\zeta} \nabla_{q} f\right) \quad \text { in } \Omega_{t} \times B\left(0, q_{m}\right)
\end{aligned}
$$

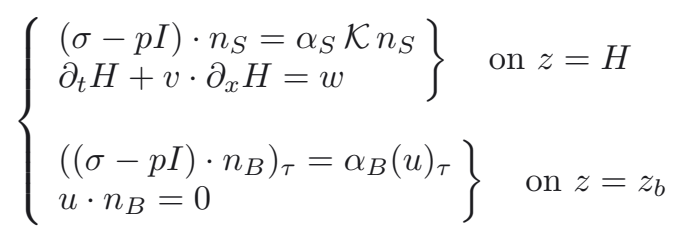

with $\Omega_{t}=\left\{(x, z): x \in \mathbb{T}, \quad z_{b}<z<H(t, x)\right\}$ and the constants $\kappa, \theta, \zeta, \mathcal{H}, q_{m}$ defined in the previous part. We indicates by $\mathbb{T}$ the assumption of horizontal periodic condition. In the boundary conditions, $I$ denotes the bi-dimensional identity tensor. We consider the surface tension coefficient $\alpha_{S}$ and the friction coefficient $\alpha_{B}$. We denote $n_{B}$ the normal vector to the bottom, $\mathcal{K}=\operatorname{div}\left(n_{S}\right)$ the mean curvature with $n_{S}$ the normal vector to the surface. By subscript $\tau$, we denote the tangential component of a vector field.

\subsection{The asymptotic shallow-water type systems}

Low Deborah number regime: In this part, we provide a shallow-water type system corresponding to the Case 1 with radial hypothesis at the leading order. Through an appropriate asymptotic expansion up to order 2 we obtain the following asymptotic system:

$$
\begin{gathered}
\partial_{t} \tilde{h}+\partial_{x}(\tilde{h} \bar{v})=0 ; \\
\rho\left(\partial_{t}(\tilde{h} \bar{v})+\partial_{x}\left(\tilde{h} \bar{v}^{2}+\frac{1}{2} g \tilde{h}^{2}\right)\right)-4 \mu \partial_{x}\left(\tilde{h} \partial_{x} \bar{v}\right) \\
+\alpha_{B} \xi \bar{v}-\alpha_{S} \tilde{h} \partial_{x}^{3}\left(z_{b}+\tilde{h}\right)+\rho g \tilde{h} \partial_{x} z_{b}=0 ;
\end{gathered}
$$

with

$$
\xi=\left(1+\frac{\alpha_{B}}{\tilde{h}^{2}} \int_{z_{b}}^{z_{b}+\tilde{h}} \int_{z_{b}}^{z} \frac{\tilde{h}-\left(\chi-z_{b}\right)}{\mu+\tau\left(n_{0}(\chi)\right)} \mathrm{d} \chi \mathrm{d} z\right)^{-1} \text { and } \tau\left(n_{0}\right)=\overline{\gamma_{1}} n_{0} \mathcal{D} e
$$

where $\bar{v}$ is the averaged horizontal velocity given by $\bar{v}=\left(\int_{z_{b}}^{z_{b}+\tilde{h}} \tilde{v}\right) / \tilde{h}$ with $\tilde{h}$ and $\tilde{v}$ defined through (4.31). The polymer density $n_{0}$ is the solution of the next $3 \mathrm{~d}$ transport equation:

$$
\partial_{t} n_{0}+u_{0} \cdot \nabla n_{0}=0
$$

defined over a time-variable vertical domain and with velocity $u_{0}=\left(\bar{v}, w_{0}\right)$; where thanks to the incompressibility equation,

$$
w_{0}=\bar{v} \partial_{x} z_{b}-\left(z-z_{b}\right) \partial_{x} \bar{v} .
$$

Thus, the unknowns of the system are $\tilde{h}, \bar{v}$ and $n_{0}$. Note that to be consistent with (3.1) the dimension of $\gamma_{1}$ is $\overline{\gamma_{1}}=\rho V_{*} L_{*} \gamma_{1}$ with $\gamma_{1}$ defined by $(4.25)$.

Intermediate Deborah number regime: In this part, we provide a shallow-water type system corresponding respectively to Case 2. It involves a more complex coupling than in Case 1 since it requires to solve an averaged 
system coupled with a full non-stationary Fokker-Planck equation. Since formal proofs are more simple than in previous part, we will just give the main steps in Section 5.

In this case we find the following asymptotic limit

$$
\begin{aligned}
& \partial_{t} h_{0}+\partial_{x}\left(h_{0} \overline{v_{0}}\right)=0 ; \\
& \rho\left(\partial_{t}\left(h_{0} \overline{v_{0}}\right)+\partial_{x}\left(h_{0}{\overline{v_{0}}}^{2}+\frac{1}{2} g h_{0}^{2}\right)\right)-4 \mu \partial_{x}\left(h_{0} \partial_{x} \overline{v_{0}}\right)+\alpha_{B} \overline{v_{0}}=\partial_{x} \int_{0}^{h_{0}}\left(\sigma_{P 0}^{11}-\sigma_{P 0}^{22}\right) ;
\end{aligned}
$$

where $\left(h_{0}, \overline{v_{0}}\right)$ are main order term in expansion of $(h, v)$ and $\left(\sigma_{P 0}, f_{0}\right)$ are coupled through the following system: for all $t \in[0, T]$, in $\Omega_{t}$

$$
\sigma_{P 0}=n_{p} \int_{\mathcal{B}}\left(\frac{\mathcal{H} q}{1-\frac{q^{2}}{q_{m}^{2}}} \otimes q\right) f_{0}(q)-\kappa \theta n_{p} \int_{\mathcal{B}} f_{0}(q) \mathrm{Id}
$$

and in $\Omega_{t} \times \mathcal{B}$ with $\mathcal{B}=B\left(0, q_{m}\right)$ :

$$
\partial_{t} f_{0}+\overline{v_{0}} \cdot \partial_{x} f_{0}-z \partial_{x} \overline{v_{0}} \partial_{z} f_{0}=-\nabla_{q} \cdot\left(\left(\begin{array}{l}
\partial_{x} \overline{v_{0}}-\frac{1}{\mu} \sigma_{P 0}^{12} \\
0-\partial_{x} \overline{v_{0}}
\end{array}\right) \cdot q f_{0}-\frac{2}{\zeta} \frac{\mathcal{H} q}{1-\frac{q^{2}}{q_{m}^{2}}} f_{0}-\frac{2 \kappa \theta}{\zeta} \nabla_{q} f_{0}\right) .
$$

This gives a viscoelastic model of thin film fluids, for polymeric flows with intermediate Deborah number. In spite of the nonlinear nature of this model, it collects the interesting case when the material has an important elastic component (intermediate Deborah number). For these fluids, the forces coming from the non-Newtonian nature affect the flow behaviour in a stronger way than in Case 1.

This system also extends the result in [10] since it contains the time derivative in the velocity field equation. The main difference is that here we deduce a shallow-water system to determine the velocity so we keep its evolution in time. In [10] a Reynolds equation is considered to define the hydrodynamic of the fluid, thus, the model reduces to a single non-linear partial differential equation in terms of the thickness of the fluid layer and the velocity field is determined through a linear equation of this thickness. See for instance the very nice paper by Oron et al. in [33] for complete description of lubrication systems for Newtonian flows in thin domain. Furthermore, in [10] the velocity is a given function for the Fokker-Planck equation, so the hydrodynamic and the rheological part are treated separately. In our case, on the other hand, a coupled problem in velocity is obtained.

Note that our system is closed to the one obtained in [5]. In this paper it is taken into account just the viscosity due to the presence of the elastically deformable particles in the fluid, or equivalently, due to the polymer presence. Thus, if we take $\mu=0$ and $\alpha_{B}=0$ in (2.16) the equation for the velocity coincides with those in [5] for flat bottom. Saving the difference that - as we mention in the Introduction - the definition of the stress tensor comes from a different model (Upper-Convected Maxwell model).

\section{Adimensionnalization}

To make the dimensionless of the equations, we first define the ratio of the thin layer $\varepsilon=\frac{H_{*}}{L_{*}}$, with $H_{*}$ and $L_{*}$ the characteristic height and length respectively, and then we take the following characteristic variables:

$$
\begin{aligned}
& x=L_{*} \tilde{x}, \quad z=H_{*} \tilde{z}, \quad v=V_{*} \tilde{v}, \quad w=\varepsilon V_{*} \tilde{w}, t=\frac{L_{*}}{V_{*}} \tilde{t}, \\
& p=\rho V_{*}^{2} \tilde{p}, \quad \alpha_{B}=\rho V_{*} \tilde{\alpha}_{B}, \alpha_{S}=\rho \frac{V_{*}^{2} L_{*}}{\varepsilon} \tilde{\alpha}_{S}, \mathcal{K}=\frac{\varepsilon}{L_{*}} \tilde{\mathcal{K}} \\
& \sigma_{P}=\rho V_{*}^{2} \tilde{\sigma_{P}}, q=Q_{*} \tilde{q}, \quad \lambda=\rho V_{*}^{2} \tilde{\lambda}
\end{aligned}
$$

with $\lambda=\kappa \theta n_{p}$ a coefficient appearing in the stress tensor $\sigma_{P}$, see (2.4). 
We define as usual the Reynolds and the Froude numbers: $\operatorname{Re}=\frac{\rho V_{*} L_{*}}{\mu}, \operatorname{Fr}=\frac{V_{*}}{\sqrt{g H_{*}}}$ and we also consider the following adimensional parameters related to the Fokker-Planck equation:

$$
\mathcal{D} e=\frac{\zeta V_{*}}{4 L_{*} \mathcal{H}}, \quad \delta=\frac{q_{m}^{2}}{Q_{*}^{2}}, \quad b=\frac{\mathcal{H} q_{m}^{2}}{\kappa \theta} .
$$

The parameter $\mathcal{D} e$ is called the Deborah number and is an indicator of how fluid a material is. In this sense, the more smaller $\mathcal{D} e$ is, the more fluid the material becomes. Its expression represents the ratio between a characteristic relaxation time for the fluid $\left(\frac{\zeta}{4 \mathcal{H}}\right)$ and the convective time scale $\left(T_{*}=\frac{L_{*}}{V_{*}}\right)$ and measures the relative importance between elastic and convective effects. The parameter $\sqrt{\delta}$ is the dimensionless maximum elongation. According to [12,34], the parameter $\delta$ is roughly the number of monomer units represented by a bead; thus it is generally larger than 10 . Finally, $b$ usually appears in the kinetic theory and it is related to microscopic constant times. In particular it is the ratio between the characteristic microscopic time related to the elasticity of the dumbbell $\left(\frac{\zeta q_{m}^{2}}{4 \kappa \theta}\right)$ and the characteristic microscopic time related to the elastic property of the fluid $\left(\frac{\zeta}{4 \mathcal{H}}\right)$-introduced before to define De number-, (see $[9,24]$ for more details on this issue).

Remark 3.1. Notice that the coefficient $\lambda=\kappa \theta n_{p}$ has the dimension assumed in (3.1), $\left[\rho V_{*}^{2}\right]$. Effectively, in the IS the units are $[\lambda]=\frac{\mathrm{Joule}}{\mathrm{m}^{3}}=\frac{\mathrm{kg}}{\mathrm{m} \cdot \mathrm{s}^{2}}$, that is exactly the dimension of $\left[\rho V_{*}^{2}\right]=\frac{\mathrm{kg}}{\mathrm{m} \cdot \mathrm{s}^{2}}$.

\subsection{Adimensional microscopic description}

Next we are going to find the dimensionless Fokker-Planck equation for the FENE model. First note that since $q_{m}$ is the maximum dumbbell extension, then we take $\mathcal{B}=B\left(0, q_{m}\right)$. Taking into account (3.1)-(3.2) the adimensional Fokker-Planck equation reads:

$$
\partial_{t} f+u \cdot \nabla f=-\nabla_{q} \cdot\left(\left(\begin{array}{ll}
\partial_{x} v & \frac{1}{\varepsilon} \partial_{z} v \\
\varepsilon \partial_{x} w & \partial_{z} w
\end{array}\right) \cdot q f-\frac{1}{2 \mathcal{D} e} F(q) f-\frac{1}{2 \mathcal{D} e} \frac{\delta}{b} \nabla_{q} f\right),
$$

where now $(t, x, q) \in \mathbb{R}^{+} \times \Omega \times B(0, \sqrt{\delta})$ and the dimensionless expression for the FENE connector reads

$$
F(q)=\frac{q}{1-\frac{q^{2}}{\delta}}
$$

Similarly we can write the following expression for the stress tensor $\sigma_{P}$ :

$$
\sigma_{P}(t, x)=\lambda\left(\frac{b}{\delta}\langle F(q) \otimes q\rangle-n \mathrm{Id}\right),
$$

where now we introduce the $q$-average $\langle\varphi\rangle=\int_{B(0, \sqrt{\delta})} \varphi(q) f(q) \mathrm{d} q$ for any function $\varphi$ and $n=\langle 1\rangle=$ $\int_{B(0, \sqrt{\delta})} f(q) \mathrm{d} q$ the density of the polymer chains. As we introduced before, the coefficient $\lambda$ is $\lambda=\kappa \theta n_{p}$. So, according to (3.1) the dimensionless stress tensor is given by:

$$
\sigma_{P}(t, x)=\tilde{\lambda}\left(\frac{b}{\delta}\langle F(q) \otimes q\rangle-n \mathrm{Id}\right) .
$$

To simplify the Fokker-Planck equation (3.3) we assume that $b \geq 1$ and $b=\delta$ in order to get the same coefficient in last two terms; similar assumptions can be found in [10,24,27], for example. So finally the adimensional Fokker-Planck equation reads as follows:

$$
\partial_{t} f+u \cdot \nabla f=-\nabla_{q} \cdot\left(\left(\begin{array}{ll}
\partial_{x} v & \frac{1}{\varepsilon} \partial_{z} v \\
\varepsilon \partial_{x} w & \check{\partial}_{z} w
\end{array}\right) \cdot q f-\frac{1}{2 \mathcal{D} e}\left(\frac{q}{1-\frac{q^{2}}{\delta}} f+\nabla_{q} f\right)\right),
$$


and the stress tensor $\sigma_{P}$ as:

$$
\sigma_{P}(t, x)=\tilde{\lambda}\left(\left\langle\frac{q}{1-\frac{q^{2}}{\delta}} \otimes q\right\rangle-n \mathrm{Id}\right) .
$$

Remark 3.2. From the adimensional FENE model above, we can find the spring potential energy for what $F(q)=\nabla_{q} P(q)$ given by:

$$
P(q)=-\frac{\delta}{2} \ln \left(1-\frac{q^{2}}{\delta}\right) .
$$

Thus, if we define the normalized "Maxwellian" function as:

$$
M(q)=\frac{1}{J_{M}}\left(1-\frac{q^{2}}{\delta}\right)^{\frac{\delta}{2}} \quad \text { with } J_{M}=\int_{B(0, \sqrt{\delta})}\left(1-\frac{q^{2}}{\delta}\right)^{\frac{\delta}{2}} \mathrm{~d} q,
$$

we have that $F(q)=-\nabla_{q}(\ln M(q))$. This allows us to write the last two terms in equation (3.6) as follows:

$$
F(q) f+\nabla_{q} f=M(q) \nabla_{q}\left(\frac{f}{M(q)}\right) .
$$

So we can also write the Fokker-Planck equation as:

$$
\partial_{t} f+u \cdot \nabla f=-\nabla_{q} \cdot\left(\left(\begin{array}{ll}
\partial_{x} v & \frac{1}{\varepsilon} \partial_{z} v \\
\varepsilon \partial_{x} w & \partial_{z} w
\end{array}\right) \cdot q f\right)+\frac{1}{2 \mathcal{D} e} \nabla_{q} \cdot\left(M(q) \nabla_{q}\left(\frac{f}{M(q)}\right)\right) \cdot
$$

\subsection{Adimensional macroscopic equations/boundary conditions}

First we develop the equations (2.7) and the boundary conditions (2.10) for each component of the velocity. By assuming a symmetric stress tensor $\sigma=\left(\begin{array}{cc}\sigma^{11} & \sigma^{12} \\ \sigma^{12} & \sigma^{22}\end{array}\right)$, the equations read:

$$
\begin{gathered}
\rho\left(\partial_{t} v+v \partial_{x} v+w \partial_{z} v\right)+\partial_{x} p=\partial_{z} \sigma^{12}+\partial_{x} \sigma^{11} \\
\rho\left(\partial_{t} w+v \partial_{x} w+w \partial_{z} w\right)+\partial_{z} p=\partial_{x} \sigma^{12}+\partial_{z} \sigma^{22}-\rho g \\
\partial_{x} v+\partial_{z} w=0
\end{gathered}
$$

and the boundary conditions:

1. Free surface

We take $n_{S}=\frac{1}{\sqrt{1+\left|\partial_{x} H\right|^{2}}}\left(\begin{array}{c}-\partial_{x} H \\ 1\end{array}\right)$, so the tension condition reads:

$$
\begin{aligned}
& -\partial_{x} H\left(\sigma^{11}-p\right)+\sigma^{12}=-\alpha_{S} \mathcal{K} \partial_{x} H \\
& -\partial_{x} H \sigma^{12}+\left(\sigma^{22}-p\right)=\alpha_{S} \mathcal{K}
\end{aligned}
$$

and the kinematic condition:

$$
\partial_{t} H+v \partial_{x} H=w
$$

2. Bottom

We take $n_{B}=\frac{1}{\sqrt{1+\left|\partial_{x} z_{b}\right|^{2}}}\left(\begin{array}{c}-\partial_{x} z_{b} \\ 1\end{array}\right)$ and the tangent vector as $\tau=\frac{1}{\sqrt{1+\left|\partial_{x} z_{b}\right|^{2}}}\left(\begin{array}{c}1 \\ \partial_{x} z_{b}\end{array}\right)$. So the friction condition reads:

$$
\partial_{x} z_{b}\left(\sigma^{11}-\sigma^{22}\right)+\left(\left|\partial_{x} z_{b}\right|^{2}-1\right) \sigma^{12}=-\alpha_{B}\left(v+w \partial_{x} z_{b}\right) \sqrt{1+\left|\partial_{x} z_{b}\right|^{2}},
$$

and the no penetration condition:

$$
-v \partial_{x} z_{b}+w=0
$$


For the development below we use the expression of $\sigma_{S}(2.3)$ and we denote $\sigma_{P}=\left(\begin{array}{c}\sigma_{P}^{11} \\ \sigma_{P}^{12} \\ \sigma_{P}^{12} \\ \sigma_{P}^{22}\end{array}\right)$. Taking into account (3.1), we write the non dimensional equations as follows (we drop the "tilde" for the sake of simplicity in notation):

$$
\begin{gathered}
\partial_{t} v+v \partial_{x} v+w \partial_{z} v+\partial_{x} p=\frac{1}{\epsilon} \partial_{z} \sigma_{P}^{12}+\partial_{x} \sigma_{P}^{11}+\frac{1}{\operatorname{Re}}\left(\frac{1}{\epsilon^{2}} \partial_{z}^{2} v+\partial_{z}\left(\partial_{x} w\right)+2 \partial_{x}^{2} v\right) \\
\partial_{t} w+v \partial_{x} w+w \partial_{z} w+\frac{1}{\epsilon^{2}} \partial_{z} p= \\
=\frac{1}{\epsilon} \partial_{x} \sigma_{P}^{12}+\frac{1}{\epsilon^{2}} \partial_{z} \sigma_{P}^{22}-\frac{1}{\epsilon^{2}} \frac{1}{\operatorname{Fr}^{2}}+\frac{1}{\operatorname{Re}}\left(\frac{1}{\epsilon^{2}}\left(\partial_{x}\left(\partial_{z} v\right)+2 \partial_{z}^{2} w\right)+\partial_{x}^{2} w\right) \\
\partial_{x} v+\partial_{z} w=0
\end{gathered}
$$

and the boundary conditions:

1. Free surface:

$$
\begin{array}{r}
\frac{1}{\epsilon} \frac{1}{\operatorname{Re}} \partial_{z} v-\varepsilon \frac{1}{\operatorname{Re}}\left(\partial_{x} H\left(2 \partial_{x} v\right)-\partial_{x} w\right)-\varepsilon \partial_{x} H\left(\sigma_{P}^{11}-p\right)+\sigma_{P}^{12}=-\varepsilon \alpha_{S} \mathcal{K} \partial_{x} H \\
-\frac{1}{\operatorname{Re}}\left(\partial_{x} H \partial_{z} v-2 \partial_{z} w\right)-\varepsilon^{2} \frac{1}{\operatorname{Re}} \partial_{x} H \partial_{x} w-\varepsilon \partial_{x} H \sigma_{P}^{12}+\sigma_{P}^{22}-p=\alpha_{S} \mathcal{K} \\
\partial_{t} H+v \partial_{x} H=w
\end{array}
$$

2. Bottom:

$$
\begin{array}{r}
-\frac{1}{\epsilon} \frac{1}{\operatorname{Re}} \partial_{z} v-\sigma_{p}^{12}+\varepsilon \frac{1}{\operatorname{Re}}\left(-\partial_{x} w+\left|\partial_{x} z_{b}\right|^{2} \partial_{z} v+2 \partial_{x} z_{b}\left(\partial_{x} v-\partial_{z} w\right)\right)+\varepsilon \partial_{x} z_{b}\left(\sigma_{P}^{11}-\sigma_{P}^{22}\right) \\
+\varepsilon^{2}\left|\partial_{x} z_{b}\right|^{2} \sigma_{P}^{12}+\varepsilon^{3} \frac{1}{\operatorname{Re}}\left|\partial_{x} z_{b}\right|^{2} \partial_{x} w=-\alpha_{B}\left(v+\varepsilon^{2} w \partial_{x} z_{b}\right) \sqrt{1+\varepsilon^{2}\left|\partial_{x} z_{b}\right|^{2}} \\
-v \partial_{x} z_{b}+w=0
\end{array}
$$

\section{LOW DEBORAH NUMBER REGIME}

\subsection{Range of coefficients and system to be studied}

Let us consider the following relations between the adimensional numbers:

$$
\frac{1}{\operatorname{Re}}=\varepsilon \mu_{0}, \quad \alpha_{S}=\varepsilon \alpha_{0 S}, \quad \alpha_{B}=\varepsilon \alpha_{0 B}, \quad \mathcal{D} e=\varepsilon \mathcal{D} e_{0}
$$

with $\mu_{0}, \alpha_{0 S}, \alpha_{0 B}, \mathcal{D} e_{0}$ of order of the unity and let us perform asymptotic expansions up to second order with respect to $\varepsilon$. Such calculations have been perfomed for the Navier-Stokes equations with free surface namely with $\sigma_{P}=0$ in $[15,32]$ justifying a corrected shallow-water system. Here the main novelty is to investigate the microscopic effect due to the Fokker-Planck equation. We will get an influence at same order than the one obtained in $[15,32]$ : the miscroscopic effect is then comparable to a friction drag term. Before writing the full system with the boundary conditions, we focus on the asymptotic Fokker-Planck equation. Remind that we need the solution $f$ to find $\sigma_{P}$ and then to solve Navier-Stokes equations.

The Fokker-Planck equation. The influence of the macroscopic flow on the polymers comes from the presence of the velocity $u$ in the Fokker-Planck equation. Denoting

$$
\nabla u=\frac{1}{\epsilon} \underbrace{\left(\begin{array}{rr}
0 & \partial_{z} v \\
0 & 0
\end{array}\right)}_{C}+\underbrace{\left(\begin{array}{lr}
\partial_{x} v & 0 \\
0 & \partial_{z} w
\end{array}\right)}_{G}+\varepsilon \underbrace{\left(\begin{array}{ll}
0 & 0 \\
\partial_{x} w & 0
\end{array}\right)}_{E},
$$


we can write equation (3.6) into the compact form

$$
T f+\tilde{B} f=\frac{1}{\epsilon} \tilde{A} f
$$

where

$$
\begin{aligned}
& T f=\partial_{t} f+v \partial_{x} f+w \partial_{z} f, \\
& \tilde{B} f=\nabla_{q} \cdot((G+\varepsilon E) q f), \quad \tilde{A} f=\nabla_{q} \cdot\left(\nabla_{q} f+F(q) f-2 \mathcal{D} e_{0} C q f\right) /\left(2 \mathcal{D} e_{0}\right) .
\end{aligned}
$$

Coupled system and boundary conditions. In conclusion, the system under consideration to perform the asymptotic analysis with respect to the adimensional number $\varepsilon$, reads:

$$
\begin{gathered}
\partial_{t} v+v \partial_{x} v+w \partial_{z} v+\partial_{x} p=\frac{1}{\epsilon} \partial_{z} \sigma_{P}^{12}+\partial_{x} \sigma_{P}^{11}+\mu_{0}\left(\frac{1}{\epsilon} \partial_{z}^{2} v+\varepsilon\left(\partial_{z}\left(\partial_{x} w\right)+2 \partial_{x}^{2} v\right)\right) \\
\partial_{t} w+v \partial_{x} w+w \partial_{z} w+\frac{1}{\epsilon^{2}} \partial_{z} p=\frac{1}{\epsilon} \partial_{x} \sigma_{P}^{12}+\frac{1}{\epsilon^{2}} \partial_{z} \sigma_{P}^{22} \\
+\mu_{0}\left(\frac{1}{\epsilon}\left(\partial_{x}\left(\partial_{z} v\right)+2 \partial_{z}^{2} w\right)+\varepsilon \partial_{x}^{2} w\right)-\frac{1}{\epsilon^{2}} \frac{1}{\mathrm{Fr}^{2}} \\
\partial_{x} v+\partial_{z} w=0
\end{gathered}
$$

with

$$
T f+\tilde{B} f=\frac{1}{\varepsilon} \tilde{A} f \text { where } T, \widetilde{A} \text { and } \widetilde{B} \text { defined by (4.4) and (4.5) }
$$

and the boundary conditions on the surface

$$
z=H\left\{\begin{array}{l}
\frac{1}{\epsilon} \mu_{0} \partial_{z} v+\frac{1}{\epsilon} \sigma_{P}^{12}-\varepsilon \mu_{0}\left(2 \partial_{x} H \partial_{x} v-\partial_{x} w\right)-\partial_{x} H\left(\sigma_{P}^{11}-p\right)=-\varepsilon \alpha_{0 S} \mathcal{K} \partial_{x} H \\
-\mu_{0}\left(\partial_{x} H \partial_{z} v-2 \partial_{z} w\right)-\partial_{x} H \sigma_{P}^{12}+\frac{1}{\epsilon}\left(\sigma_{P}^{22}-p\right)-\varepsilon^{2} \mu_{0} \partial_{x} H \partial_{x} w=\alpha_{0 S} \mathcal{K} \\
\partial_{t} H+v \partial_{x} H=w
\end{array}\right.
$$

and on the bottom

$$
z=z_{b}\left\{\begin{aligned}
- & \frac{1}{\epsilon} \mu_{0} \partial_{z} v-\frac{1}{\epsilon} \sigma_{P}^{12}+\partial_{x} z_{b}\left(\sigma_{P}^{11}-\sigma_{P}^{22}\right) \\
& +\varepsilon \mu_{0}\left(-\partial_{x} w+\left|\partial_{x} z_{b}\right|^{2} \partial_{z} v+2 \partial_{x} z_{b}\left(\partial_{x} v-\partial_{z} w\right)\right) \\
& +\varepsilon\left|\partial_{x} z_{b}\right|^{2} \sigma_{P}^{12}+\varepsilon^{3} \mu_{0}\left|\partial_{x} z_{b}\right|^{2} \partial_{x} w=-\alpha_{0 B}\left(v+\varepsilon^{2} w \partial_{x} z_{b}\right) \sqrt{1+\varepsilon^{2}\left|\partial_{x} z_{b}\right|^{2}} \\
- & v \partial_{x} z_{b}+w=0 .
\end{aligned}\right.
$$

\subsection{Pressure up to order one/integrated horizontal components}

Let us integrate equations (4.6)-(4.8) with respect to the vertical variable to prepare things in order to get a shallow-water type system.

Pressure expression. We obtain the expression for the pressure by integrating equation (4.7) from $z$ to $H$. Note that we just take into account terms of order $\varepsilon^{0}$ and $\varepsilon$ :

$$
p(z)=-\frac{1}{\operatorname{Fr}^{2}}(z-H)+\sigma_{P}^{22}-\varepsilon \partial_{x} \int_{z}^{H} \sigma_{P}^{12}+\varepsilon \mu_{0} \partial_{z} w+\left[-\left(\sigma_{P}^{22}-p\right)+\varepsilon \sigma_{P}^{12} \partial_{x} H-\varepsilon \mu_{0} \partial_{z} w\right]_{\mid z=H}+\mathcal{O}\left(\varepsilon^{2}\right)
$$

and thanks to second condition in (4.10), it reads:

$$
p(z)=-\frac{1}{\operatorname{Fr}^{2}}(z-H)+\sigma_{P}^{22}-\varepsilon \partial_{x} \int_{z}^{H} \sigma_{P}^{12}+\varepsilon \mu_{0} \partial_{z} w-\varepsilon\left[\alpha_{0 S} \mathcal{K}+\mu_{0} \partial_{x} H \partial_{z} v-\mu_{0} \partial_{z} w\right]_{\mid z=H}+\mathcal{O}\left(\varepsilon^{2}\right) .
$$


Height equation. As usually, we integrate equation (4.8) from $z_{b}$ to $H$. This gives

$$
\partial_{t} H+\partial_{x} \int_{z_{b}}^{H} v \mathrm{~d} z=0
$$

Vertical average of horizontal momentum equation. Let us integrate equation (4.6) from $z_{b}$ to $H$ using first conditions in (4.10) and (4.11). We get the following equation

$$
\begin{aligned}
& \partial_{t} \int_{z_{b}}^{H} v \mathrm{~d} z+\partial_{x} \int_{z_{b}}^{H} v^{2} \mathrm{~d} z+\partial_{x} \int_{z_{b}}^{H} p \mathrm{~d} z+p\left(z_{b}\right) \partial_{x} z_{b} \\
& =\partial_{x} \int_{z_{b}}^{H} \sigma_{P}^{11} \mathrm{~d} z+2 \varepsilon \mu_{0} \partial_{x} \int_{z_{b}}^{H} \partial_{x} v \mathrm{~d} z-\varepsilon \alpha_{0 S} \mathcal{K} \partial_{x} H-\alpha_{0 B} v_{\mid z=z_{b}} \\
& \quad+\partial_{x} z_{b} \sigma_{P}^{22}{ }_{\mid z=z_{b}}-\varepsilon\left[\mu_{0}\left|\partial_{x} z_{b}\right|^{2} \partial_{z} v-2 \mu_{0} \partial_{x} z_{b} \partial_{z} w+\left|\partial_{x} z_{b}\right|^{2} \sigma_{P}^{12}\right]_{\mid z=z_{b}}+\mathcal{O}\left(\varepsilon^{2}\right) .
\end{aligned}
$$

\subsection{Second order shallow-water type approximation}

As in $[15,32]$, we want to obtain the viscous effects so we must achieve the second order approximation. Let us expand the unknowns in terms of $\varepsilon$, namely:

$$
\begin{aligned}
& v=v_{0}+\varepsilon v_{1}+\mathcal{O}\left(\varepsilon^{2}\right), \\
& w=w_{0}+\varepsilon w_{1}+\mathcal{O}\left(\varepsilon^{2}\right), \\
& p=p_{0}+\varepsilon p_{1}+\mathcal{O}\left(\varepsilon^{2}\right), \\
& h=h_{0}+\varepsilon h_{1}+\mathcal{O}\left(\varepsilon^{2}\right), \\
& f=f_{0}+\varepsilon f_{1}+\mathcal{O}\left(\varepsilon^{2}\right) .
\end{aligned}
$$

So, $\sigma_{P}=\sigma_{P 0}+\varepsilon \sigma_{P 1}+\mathcal{O}\left(\varepsilon^{2}\right)$ where $\sigma_{P 0}$ and $\sigma_{P 1}$ correspond to (3.7) for $f_{0}$ and $f_{1}$ respectively. If we write the equations at main order $1 / \varepsilon$, we get:

$$
\mu_{0} \partial_{z}^{2} v_{0}=-\partial_{z} \sigma_{P 0}^{12}, \quad \mu_{0} \partial_{z} v_{0 \mid z=H}=-\sigma_{P 0 \mid z=H}^{12}, \quad \mu_{0} \partial_{z} v_{0 \mid z=z_{b}}=-\sigma_{P 0 \mid z=z_{b}}^{12} .
$$

The classical way ( $c f$. [15]) to deduce the hydrostatic system is based on the fact that the velocity $v$ does not depend on $z$ up to first order, so $v_{0}=v_{0}(t, x)$ and $\partial_{z} v_{0}=0$. But with the previous equations we find the velocity $v_{0}$ may depend on the stress tensor $\sigma_{P}$ through the expression above.

In the sequel, we will split our study in two parts. In a first part, we will assume some radial hypothesis at the leading order which help to simplify the study and give explicit calculation of the microscopic effect. In a second part, we give the coupling system.

\section{Radial hypothesis: Explicit drag effect through a nonlocal term}

In this part, we assume radial properties over the probability density function at the leading order $f_{0}$, which help to conclude that $\sigma_{P 0}^{12}=0$ and therefore that $\partial_{z} v_{0}=0$ through the system above. We know that $\sigma_{P}$ comes from the solution of the Fokker-Planck equation according to (3.7). So we focus on solve (4.9) taking into account that $\mu_{0} \partial_{z} v_{0}=-\sigma_{P 0}^{12}$. This is the subject of this section where we will find the expression of $\sigma_{P}$ and in particular we will obtain that $\sigma_{P 0}^{12}=0$. Hence $\partial_{z} v_{0}=0$ as in [15]. Then we perform the ansatz up to order two and get a correction in the drag term for the shallow-water system.

First we must prove the existence of the solution of the equation (4.9) since we have changed the original Fokker-Planck equation in order to introduce the asymptotic hypotheses. Besides, we must also take into account the restriction on $v_{0}$ with respect to $\sigma_{P 0}^{12}$. We find the next result:

Proposition 4.1. Let $H_{M_{\text {rad }}}$ the space of radial functions in $H_{M}=\left\{f / \frac{f}{\sqrt{M}} \in L^{2}(\mathcal{B})\right\}$ defined as follows:

$$
H_{M_{\text {rad }}}=\left\{g \in H_{M} / g=G(|q|) \text { for some operator } G\right\} .
$$


We consider the matrix $C$ defined as

$$
C=\left(\begin{array}{rr}
0 & \partial_{z} v \\
0 & 0
\end{array}\right)
$$

with $\partial_{z} v=-\frac{1}{\mu_{0}} \sigma_{P}^{12}$ and being

$$
\sigma_{P}^{i j}=\tilde{\lambda}\left(\int_{\mathcal{B}} \frac{q_{i} q_{j}}{1-\frac{q^{2}}{\delta}} f(q) \mathrm{d} q-\int_{\mathcal{B}} f(q) \mathrm{d} q \delta_{i j}\right)
$$

with $q=\left(q_{1}, q_{2}\right), \mathcal{B}=B(0, \sqrt{\delta})$ and $\delta_{i j}$ the Kronecker delta. Then the equation $\tilde{A} f=0$, that reads

$$
\nabla_{q} \cdot\left[M \nabla_{q}\left(\frac{f}{M}\right)-2 \mathcal{D} e_{0} C q f\right]=0
$$

admits a unique solution $f \in H_{M_{\text {rad }}}$ and it is of the form

$$
f=a_{0} M(q)
$$

for some constant $a_{0} \in \mathbb{R}$ and $M(q)$ defined in (3.8).

Proof. First we remark that $\sigma_{P}^{12}=0$. Indeed, if we solve the integral by using the polar variables:

$$
\left\{\begin{array}{l}
q_{1}=r \cos (\theta) \\
q_{2}=r \sin (\theta)
\end{array} \quad \text { with } r \in(0, \sqrt{\delta}), \theta \in(0,2 \pi),\right.
$$

we obtain

$$
\sigma_{P}^{12}=\tilde{\lambda} \int_{\mathcal{B}} \frac{q_{1} q_{2}}{1-\frac{q^{2}}{\delta}} f(q) \mathrm{d} q=\tilde{\lambda} \int_{0}^{\sqrt{\delta}} \int_{0}^{2 \pi} \frac{r^{3} \sin (\theta) \cos (\theta)}{1-\frac{r^{2}}{\delta}} f(r, \theta) \mathrm{d} \theta \mathrm{d} r
$$

but if we search $f \in H_{M_{\text {rad }}}$ we have $f(q)=f(r, \theta)=f(r)$, so we find

$$
\sigma_{P}^{12}=\tilde{\lambda} \int_{0}^{\sqrt{\delta}} \frac{r^{3}}{1-\frac{r^{2}}{\delta}} f(r) \mathrm{d} r \int_{0}^{2 \pi} \sin (\theta) \cos (\theta) \mathrm{d} \theta=0 .
$$

This makes that $\partial_{z} v=-\frac{1}{\mu_{0}} \sigma_{P}^{12}=0$ and thanks to the definition of the matrix $C$, we get $C=0$. Then it is standard that there is a unique solution

$$
f(q)=a_{0} M(q)
$$

for some constant $a_{0} \in \mathbb{R}$.

Second order approximation of the stress tensor $\sigma_{P}$. Since the stress tensor $\sigma$ depends on the density $f$ and with the objective of getting a second order model, we search now the second order approximation of $f$ collected in the following proposition.

Proposition 4.2. We consider an asymptotic expansion of $f$ and $v$ in powers of $\varepsilon$, namely:

$$
\begin{aligned}
& f=f_{0}+\varepsilon f_{1}+\mathcal{O}\left(\varepsilon^{2}\right) ; \\
& v=v_{0}+\varepsilon v_{1}+\mathcal{O}\left(\varepsilon^{2}\right),
\end{aligned}
$$

and assume that $f_{0} \in H_{M_{\text {rad }}}$. Then the solution of the Fokker-Planck equation (4.9) is approximated up to order 2 through:

$$
\begin{gathered}
f_{0}=a_{0} M(q), \\
f_{1}=\frac{1}{2} \mathcal{D} e_{0} a_{0}\left(\left(C_{1}^{s}+2 G_{0}\right) q \otimes q\right) M(q),
\end{gathered}
$$

where $a_{0}$ is the solution of the equation:

$$
\partial_{t} a_{0}+u_{0} \cdot \nabla a_{0}=0
$$


and the matrix $C_{1}^{s}+2 G_{0}$ depending on the velocity is defined as follows:

$$
C_{1}^{s}+2 G_{0}=\left(\begin{array}{rr}
\partial_{x} v_{0} & \partial_{z} v_{1} \\
\partial_{z} v_{1} & -\partial_{x} v_{0}
\end{array}\right)
$$

Proof. The proof follows the lines in [12] taking care of the small parameter and the definitions of the operators.

We must solve the Fokker-Planck equation (4.9):

$$
(T+\tilde{B}) f=\frac{1}{\varepsilon} \tilde{A} f
$$

with $T, \tilde{B}$ and $\tilde{A}$ given by (4.4)-(4.5). First of all we take into account the development of the velocity $v$ in the definitions of the matrices involved in operators $\tilde{B}$ and $\tilde{A}$ in order to get an equation with operators independent of $\varepsilon$. For example for matrix $C=\left(\begin{array}{rr}0 & \partial_{z} v \\ 0 & 0\end{array}\right)$, using the ansatz of $v$, it reads at order one:

$$
C=C_{0}+\varepsilon C_{1}+\mathcal{O}\left(\varepsilon^{2}\right) \quad \text { with } C_{0}=\left(\begin{array}{rr}
0 & \partial_{z} v_{0} \\
0 & 0
\end{array}\right) \text { and } C_{1}=\left(\begin{array}{rr}
0 & \partial_{z} v_{1} \\
0 & 0
\end{array}\right) .
$$

Thus, we write the terms up to order 1. From the 1.h.s.:

$$
(T+\tilde{B}) f=T f+\nabla_{q} \cdot((G+\varepsilon E) q f)=T_{0} f_{0}+\nabla_{q} \cdot\left(G_{0} q f_{0}\right)+\mathcal{O}(\varepsilon),
$$

with $T_{0} f_{0}=\partial_{t} f_{0}+v_{0} \partial_{x} f_{0}+w_{0} \partial_{z} f_{0}$.

Let us introduce the operator $A$ by $A f=\frac{1}{2} \nabla_{q}\left(M \nabla_{q}\left(\frac{f}{M}\right)\right)$, then for the r.h.s. we write:

$$
\begin{aligned}
\tilde{A} f=\frac{1}{\mathcal{D} e_{0}} & A f-\nabla_{q} \cdot(C q f) \\
& =\frac{1}{\mathcal{D} e_{0}} A f_{0}-\nabla_{q} \cdot\left(C_{0} q f_{0}\right)+\varepsilon\left(\frac{1}{\mathcal{D} e_{0}} A f_{1}-\nabla_{q} \cdot\left(C_{1} q f_{0}\right)-\nabla_{q} \cdot\left(C_{0} q f_{1}\right)\right)+\mathcal{O}\left(\varepsilon^{2}\right) .
\end{aligned}
$$

Then, the equation to solve reads:

$$
T_{0} f_{0}+\nabla_{q} \cdot\left(G_{0} q f_{0}\right)=\frac{1}{\varepsilon}\left(\frac{1}{\mathcal{D} e_{0}} A f_{0}-\nabla_{q} \cdot\left(C_{0} q f_{0}\right)+\varepsilon \frac{1}{\mathcal{D} e_{0}} A f_{1}-\varepsilon \nabla_{q} \cdot\left(C_{1} q f_{0}\right)-\varepsilon \nabla_{q} \cdot\left(C_{0} q f_{1}\right)\right)+\mathcal{O}(\varepsilon) .
$$

Thanks to the hypothesis $f_{0} \in H_{M_{\text {rad }}}$ and Proposition 4.1 we have that the matrix $C_{0}$ vanishes, so:

$$
T_{0} f_{0}+\nabla_{q} \cdot\left(G_{0} q f_{0}\right)=\frac{1}{\varepsilon}\left(\frac{1}{\mathcal{D} e_{0}} A f_{0}+\varepsilon \frac{1}{\mathcal{D} e_{0}} A f_{1}-\varepsilon \nabla_{q} \cdot\left(C_{1} q f_{0}\right)\right)+\mathcal{O}(\varepsilon)
$$

that can be also written as:

$$
T_{0} f_{0}+\nabla_{q} \cdot\left(\left(G_{0}+C_{1}\right) q f_{0}\right)=\frac{1}{\varepsilon}\left(\frac{1}{\mathcal{D} e_{0}} A f_{0}+\varepsilon \frac{1}{\mathcal{D} e_{0}} A f_{1}\right)+\mathcal{O}(\varepsilon) .
$$

If we denote by $K f_{0}=T_{0} f_{0}+\nabla_{q} \cdot\left(\left(G_{0}+C_{1}\right) q f_{0}\right)$ and $Q(f)=\frac{1}{\mathcal{D} e_{0}} A f$ we have to solve the following equation:

$$
K f_{0}=\frac{1}{\varepsilon}\left(Q\left(f_{0}\right)+\varepsilon Q\left(f_{1}\right)\right)+\mathcal{O}(\varepsilon)
$$

that suits in the case studied in [12]. Following this work, to find $f_{0}$ and $f_{1}$ we match the terms of same orders, obtaining that these solutions are given by:

- $A f_{0}=0$

- $\frac{1}{\mathcal{D} e_{0}} A f_{1}=(I-\Pi) K f_{0}$ 
with $\Pi$ the $L^{2}$-orthogonal projection of $H_{M}$ onto the kernel of the adjoint space $A^{*}$ (denoted by $N\left(A^{*}\right)$, that coincides with $N(A)$ because $A$ is self-adjoint on $\left.H_{M}\right)$ ( $c f$. [12]). It is defined as

$$
\Pi \varphi(q)=M(q) \int_{\mathcal{B}} \varphi(q) \mathrm{d} q,
$$

for any function $\varphi \in H_{M}$.

From the first equation we obtain directly (see Prop. 4.1) that

$$
f_{0}=a_{0} M(q) .
$$

We solve the equation in $f_{1}$, that reads:

$$
\frac{1}{\mathcal{D} e_{0}} A f_{1}=(I-\Pi)\left(T_{0} f_{0}+\nabla_{q} \cdot\left(\left(G_{0}+C_{1}\right) q f_{0}\right)\right) .
$$

By one hand we have that $\Pi\left(\nabla_{q} \cdot\left(\left(G_{0}+C_{1}\right) q f_{0}\right)\right)=0$ :

$$
\Pi\left(\nabla_{q} \cdot\left(\left(G_{0}+C_{1}\right) q f_{0}\right)\right)=M(q) \int_{\mathcal{B}} \nabla_{q} \cdot\left(\left(G_{0}+C_{1}\right) q f_{0}\right) \mathrm{d} q=0
$$

and by other hand we have since $f_{0} \in N(A)$ that $\Pi T_{0}=T_{0} \Pi$ ( $c f$. [12]), so:

$$
(I-\Pi) T_{0} f_{0}=T_{0} f_{0}-\Pi T_{0} f_{0}=T_{0} f_{0}-T_{0} \Pi f_{0}=T_{0} f_{0}-T_{0} f_{0}=0 .
$$

So the equation to solve becomes:

$$
\frac{1}{\mathcal{D} e_{0}} A f_{1}=\nabla_{q} \cdot\left(G_{0} q f_{0}\right)+\nabla_{q} \cdot\left(C_{1} q f_{0}\right) .
$$

We focus again on matrix $C_{1}=\left(\begin{array}{rr}0 & \partial_{z} v_{1} \\ 0 & 0\end{array}\right)$ that we divide in its symmetric and antisymmetric parts as follows:

$$
C_{1}=\frac{1}{2}\left(\begin{array}{lr}
0 & \partial_{z} v_{1} \\
\partial_{z} v_{1} & 0
\end{array}\right)+\frac{1}{2}\left(\begin{array}{lr}
0 & \partial_{z} v_{1} \\
-\partial_{z} v_{1} & 0
\end{array}\right)=\frac{1}{2}\left(C_{1}^{s}+C_{1}^{a s}\right)
$$

Now the term in the equation above containing $C_{1}$ can be written as:

$$
\nabla_{q} \cdot\left(C_{1} q f_{0}\right)=\frac{1}{2} \nabla_{q} \cdot\left(\left(C_{1}^{s}+C_{1}^{a s}\right) q f_{0}\right)=\nabla_{q} \cdot\left(C_{1}^{s} q f_{0}\right),
$$

because for the antisymmetric part we have:

$$
\begin{aligned}
& \nabla_{q} \cdot\left(C_{1}^{a s} q f_{0}\right)=\nabla_{q} \cdot\left[\left(\begin{array}{c}
\left(\partial_{z} v_{1}\right) q_{2} \\
-\left(\partial_{z} v_{1}\right) q_{1}
\end{array}\right) a_{0} M(q)\right]= \\
& =a_{0}\left(\begin{array}{c}
\partial_{z} v_{1} q_{2} \\
-\partial_{z} v_{1} q_{1}
\end{array}\right) \nabla_{q} M(q)=a_{0}\left(\begin{array}{c}
\partial_{z} v_{1} q_{2} \\
-\partial_{z} v_{1} q_{1}
\end{array}\right)\left(\begin{array}{c}
U(q) q_{1} \\
U(q) q_{2}
\end{array}\right)^{t}=0,
\end{aligned}
$$

being $U(q)=-\frac{1}{J}\left(1-\frac{q^{2}}{\delta}\right)^{\delta / 2-1}$. So finally we must solve:

$$
\frac{1}{\mathcal{D} e_{0}} A f_{1}=\frac{1}{2} \nabla_{q} \cdot\left(2 G_{0} q f_{0}+C_{1}^{s} q f_{0}\right) .
$$


Thanks to the definition of $A$, it suffices to seek $f_{1}$ as:

$$
\frac{1}{\mathcal{D} e_{0}} \nabla_{q}\left(\frac{f_{1}}{M}\right)=a_{0}\left(2 G_{0}+C_{1}^{s}\right) q
$$

and the solution reads

$$
f_{1}=\mathcal{D} e_{0} a_{0}\left(\left(2 G_{0}+C_{1}^{s}\right) q \cdot q\right) M(q) .
$$

This is the unique possible solution to (4.22). To obtain the constant $a_{0}$, we take $f_{0}$ to the Fokker-Planck equation and we integrate in $\mathcal{B}$ using the radial property, so we find that $a_{0}$ is solution of:

$$
\partial_{t} a_{0}+u_{0} \cdot \nabla a_{0}=0 .
$$

Remark 4.3. Notice that the radial hypothesis for $f_{0}$ is equivalent to assume that $\partial_{z} v_{0}=0$. In Proposition 4.1 we proved that if $f$ is radial, then the velocity does not depend on $z$ at main order. If we assume now that $\partial_{z} v_{0}=0$, then the matrix $C_{0}$ vanishes. Thus, the corresponding equation (4.16) reduces to

$$
\nabla_{q} \cdot\left[M \nabla_{q}\left(\frac{f_{0}}{M}\right)\right]=0
$$

but since function $M$ is radial, then $f_{0}$ must be also radial.

Remark 4.4 (About the radial hypothesis). As we explained before, the motivation of this assumption is to find $\partial_{z} v_{0}=0$. This is the usual "motion by slices" property of the shallow-water systems that is needed to develop the derivation $(c f$. [15]). The radial hypothesis is just the translation to the microscopic frame of the classical shallow flows property. Furthermore in the reference works $[10,12]$, the solution of the non-stationary Fokker-Planck equation has been studied in the case of small Deborah number. In these works the solution at the leading order is also given by $f_{0}=n_{0} M(q)$. Then this part of the probability density function does not contribute in the 1-2 component of the extra stress tensor due to the polymer chains, $\sigma_{P 0}^{12}=0$. This is also the situation that we get here under the radial hypothesis, so it is not a very restrictive assumption for the whole system.

Explicit expression of the stress tensor. Following (3.7) the stress tensor $\sigma_{P}$ is defined by:

$$
\sigma_{P}=\tilde{\lambda}(\langle F(q) \otimes q\rangle-n \mathrm{Id}),
$$

being $n=\int_{\mathcal{B}} f(q) \mathrm{d} q$. The average $\langle\cdot\rangle$ is what establish the relationship with the probability density $f$ in the next way:

$$
\langle\varphi\rangle=\int_{\mathcal{B}} \varphi f \mathrm{~d} q
$$

So taking into account the development of $f$ taken in Proposition 4.2 and that $\sigma_{P}=\sigma_{P 0}+\varepsilon \sigma_{P 1}+\mathcal{O}\left(\varepsilon^{2}\right)$, we can specify its terms as:

$$
\sigma_{P 0}=\tilde{\lambda}\left(\langle F(q) \otimes q\rangle_{0}-n_{0} \mathrm{Id}\right), \quad \sigma_{P 1}=\tilde{\lambda}\left(\langle F(q) \otimes q\rangle_{1}-n_{1} \mathrm{Id}\right) .
$$

Being $\langle\cdot\rangle_{i}$ the corresponding average by taking $f_{i}$ respectively in $(4.23)$ and so $n_{i}=\int_{\mathcal{B}} f_{i}(q) \mathrm{d} q$ for $i=0,1$. Regarding the definition of $n_{0}$ we must note that

$$
n_{0}=\int_{\mathcal{B}} f_{0}(q) \mathrm{d} q=\int_{\mathcal{B}} a_{0} M(q) \mathrm{d} q=a_{0} .
$$

So from now on we will write $f_{0}=n_{0} M(q)$. After some calculations we get:

$$
\sigma_{P 0}=\gamma_{0} n_{0}\left(\begin{array}{ll}
1 & 0 \\
0 & 1
\end{array}\right), \text { for } \gamma_{0}=\tilde{\lambda}\left(\frac{\pi \beta(3)}{J}-1\right)
$$


and

$$
\sigma_{P 1}=\gamma_{1} n_{0} \mathcal{D} e_{0}\left(\begin{array}{rr}
\partial_{x} v_{0} & \partial_{z} v_{1} \\
\partial_{z} v_{1} & -\partial_{x} v_{0}
\end{array}\right), \text { for } \gamma_{1}=\frac{\tilde{\lambda} \pi}{2 J}\left(\frac{\beta(5)}{2}-\delta^{2} \operatorname{Eul}\left(4, \frac{\delta}{2}+1\right)\right) .
$$

Note that these quantities correspond to the contribution to the friction effect due to the polymer presence into the fluid that shall be taken into account for the shallow-water model.

Remark 4.5. In previous definitions we have denoted

$$
\beta(p)=\int_{0}^{\sqrt{\delta}} r^{p}\left(1-\frac{r^{2}}{\delta}\right)^{\delta / 2-1} \mathrm{~d} r
$$

that can be solved as follows

$$
\beta(p)=\frac{1}{2} \delta^{\frac{p+1}{2}} \operatorname{Eul}\left(\frac{p+1}{2}, \frac{\delta}{2}\right), \quad \operatorname{Eul}(x, y)=\int_{0}^{1} t^{x-1}(1-t)^{y-1} \mathrm{~d} t,
$$

and we can also calculate $J$ in (3.8) by Euler function:

$$
J=\pi \delta \operatorname{Eul}\left(1, \frac{\delta}{2}+1\right)
$$

The shallow-water system. Since we have the expression of $\sigma_{P}$ up to order one, we focus now on the integrated momentum equations taking care of new terms.

First order approximation. We write equations (4.12), (4.13) and (4.19) up to first order and we obtain:

$$
\begin{gathered}
p_{0}(z)=-\frac{1}{\operatorname{Fr}^{2}}\left(z-\left(z_{b}+h_{0}\right)\right)+\sigma_{P 0}^{22} ; \\
\partial_{t} h_{0}+\partial_{x}\left(h_{0} v_{0}\right)=0 ; \\
\partial_{t} n_{0}+v_{0} \partial_{x} n_{0}+w_{0} \partial_{z} n_{0}=0,
\end{gathered}
$$

where $w_{0}$ is computed from the divergence free equation and using the boundary condition at the bottom (4.11):

$$
\left\{\begin{array}{l}
\partial_{z} w_{0}=-\partial_{x} v_{0} \\
w_{0}-v_{0} \partial_{x} z_{b}=0 \quad \text { on } z=z_{b}
\end{array}\right.
$$

so

$$
w_{0}=v_{0} \partial_{x} z_{b}-\left(z-z_{b}\right) \partial_{x} v_{0} .
$$

Now we take them to equation (4.14) and we take into account that $\sigma_{P 0}$ does not depends on $z$ (see Eq. (4.24)). Thus we get:

$$
\partial_{t}\left(h_{0} v_{0}\right)+\partial_{x}\left(h_{0} v_{0}^{2}\right)+\frac{1}{2} \frac{1}{\operatorname{Fr}^{2}} \partial_{x}\left(h_{0}^{2}\right)=-\alpha_{0 B} v_{0}-\frac{1}{\operatorname{Fr}^{2}} h_{0} \partial_{x} z_{b}
$$

As we can see in this equation neither pressure nor viscosity effects appear. We must search for the second order approximation to introduce these effects into the model.

Second order approximation. Now we consider the development in Section 4.3 for all variables up to second order and we define

$$
\tilde{v}=v_{0}+\varepsilon v_{1}, \quad \tilde{w}=w_{0}+\varepsilon w_{1}, \quad \tilde{p}=p_{0}+\varepsilon p_{1}, \quad \tilde{h}=h_{0}+\varepsilon h_{1}, \quad \tilde{f}=f_{0}+\varepsilon f_{1},
$$


together with $\tilde{\sigma}_{P}=\sigma_{P 0}+\varepsilon \sigma_{P 1}$. We also consider the second order approximation of the mean curvature to the surface $\mathcal{K}=\partial_{x}^{2}\left(z_{b}+\tilde{h}\right)+\mathcal{O}\left(\varepsilon^{2}\right)$. Equations (4.27)-(4.30) represents a shallow-water system with friction as a formal approximation in $\mathcal{O}(\varepsilon)$ of the viscous hydrostatic system, and therefore of the Navier-Stokes equations. But as has been pointed out in [15], we can improve this approximation by performing a correction of the horizontal velocity depending on $z, \tilde{v}=\tilde{v}(t, x, z)$. To do that we search for an expression for the velocity $\tilde{v}$ from equation (4.6):

$$
\frac{1}{\epsilon} \mu_{0} \partial_{z}^{2} \tilde{v}=\partial_{t} v_{0}+v_{0} \partial_{x} v_{0}+\partial_{x} p_{0}-\frac{1}{\epsilon} \partial_{z} \sigma_{P 0}^{12}-\partial_{z} \sigma_{P 1}^{12}-\partial_{x} \sigma_{P 0}^{11}+\mathcal{O}(\varepsilon) .
$$

By using (4.26), (4.30) and taking into account that $\sigma_{P 0}^{12}=0$, we have:

$$
\frac{1}{\epsilon} \mu_{0} \partial_{z}^{2} \tilde{v}=-\frac{1}{h_{0}} \alpha_{0 B} \tilde{v}_{\mid z=z_{b}}-\partial_{z} \sigma_{P 1}^{12}+\mathcal{O}(\varepsilon) .
$$

At this stage we know the expression of $\sigma_{P 1}^{12}=\tau\left(n_{0}\right) \partial_{z} v_{1}$ with $\tau\left(n_{0}\right)=\gamma_{1} n_{0} \mathcal{D} e_{0}$, so if we insert this into previous equation:

$$
\frac{1}{\epsilon} \mu_{0} \partial_{z}^{2} \tilde{v}=-\frac{1}{h_{0}} \alpha_{0 B} \tilde{v}_{\mid z=z_{b}}-\partial_{z}\left(\tau\left(n_{0}\right) \partial_{z} v_{1}\right)+\mathcal{O}(\varepsilon) .
$$

Now we write $\partial_{z} v_{1}=\partial_{z}\left(\frac{1}{\varepsilon}\left(\tilde{v}-v_{0}\right)\right)=\frac{1}{\varepsilon} \partial_{z} \tilde{v}$ since $v_{0}$ does not depend on $z$. Thus

$$
\frac{1}{\epsilon} \partial_{z}\left(\left(\mu_{0}+\tau\left(n_{0}\right)\right) \partial_{z} \tilde{v}\right)=-\frac{1}{h_{0}} \alpha_{0 B} \tilde{v}_{\mid z=z_{b}}+\mathcal{O}(\varepsilon) .
$$

Now we integrate twice from $z_{b}$ to $z$ and use the first boundary condition in (4.11) to get:

$$
\tilde{v}=\tilde{v}_{\mid z=z_{b}}+\varepsilon \frac{\alpha_{0 B}}{h_{0}} \tilde{v}_{\mid z=z_{b}} \int_{z_{b}}^{z} \frac{h_{0}-\left(\chi-z_{b}\right)}{\mu_{0}+\tau\left(n_{0}(\chi)\right)} \mathrm{d} \chi+\mathcal{O}\left(\varepsilon^{2}\right) .
$$

This expression gives a more detailed horizontal velocity through a parabolic correction. Then we obtain the average of the velocity as $\bar{v}=\frac{1}{h} \int_{z_{b}}^{z_{b}+h} \tilde{v} \mathrm{~d} z$ :

$$
\bar{v}=\xi^{-1} \tilde{v}_{\mid z=z_{b}}+\mathcal{O}\left(\varepsilon^{2}\right),
$$

where

$$
\xi=\left(1+\varepsilon \frac{\alpha_{0 B}}{h_{0}^{2}} \int_{z_{b}}^{z_{b}+\tilde{h}} \int_{z_{b}}^{z} \frac{h_{0}-\left(\chi-z_{b}\right)}{\mu_{0}+\tau\left(n_{0}(\chi)\right)} \mathrm{d} \chi \mathrm{d} z\right)^{-1}
$$

To deduce the system we must take also into account the first order terms in the pressure. So we calculate $\partial_{x} \int_{z_{b}}^{z_{b}+\tilde{h}} p \mathrm{~d} z$ from (4.12) and using (4.24) and (4.25), we find:

$$
\partial_{x} \int_{z_{b}}^{z_{b}+\tilde{h}} p \mathrm{~d} z=\frac{1}{2} \frac{1}{\operatorname{Fr}^{2}} \partial_{x}\left(\tilde{h}^{2}\right)+\partial_{x}\left(\tilde{h} \tilde{\sigma}_{P}^{22}\right)-2 \varepsilon \mu_{0} \partial_{x}\left(h_{0} \partial_{x} v_{0}\right)-\varepsilon \alpha_{0 S} \partial_{x}\left(h_{0} \partial_{x}^{2}\left(z_{b}+h_{0}\right)\right)+\mathcal{O}\left(\varepsilon^{2}\right) .
$$

We insert this equation into (4.14) and we use (4.33) and last conditions in (4.10) and (4.11) for simplifications. Dropping the $\mathcal{O}\left(\varepsilon^{2}\right)$ we finally write the system:

$$
\begin{gathered}
\partial_{t} \tilde{h}+\partial_{x}(\tilde{h} \bar{v})=0 \\
\partial_{t}(\tilde{h} \bar{v})+\partial_{x}\left(\tilde{h} \bar{v}^{2}\right)+\frac{1}{2} \frac{1}{\operatorname{Fr}^{2}} \partial_{x}\left(\tilde{h}^{2}\right)-4 \varepsilon \mu_{0} \partial_{x}\left(\tilde{h} \partial_{x} \bar{v}\right) \\
+\alpha_{0 B} \xi \bar{v}-\varepsilon \alpha_{0 S} \tilde{h} \partial_{x}^{3}\left(z_{b}+\tilde{h}\right)+\frac{1}{\operatorname{Fr}^{2}} \tilde{h} \partial_{x} z_{b}=0 ;
\end{gathered}
$$


where

$$
\xi=\left(1+\varepsilon \frac{\alpha_{0 B}}{\tilde{h}^{2}} \int_{z_{b}}^{z_{b}+\tilde{h}} \int_{z_{b}}^{z} \frac{\tilde{h}-\left(\chi-z_{b}\right)}{\mu_{0}+\tau\left(n_{0}(\chi)\right)} \mathrm{d} \chi \mathrm{d} z\right)^{-1} \text { and } \tau\left(n_{0}\right)=\gamma_{1} n_{0} \mathcal{D} e_{0} .
$$

The polymer density $n_{0}$ is the solution of the transport equation:

$$
\partial_{t} n_{0}+\bar{v} \partial_{x} n_{0}+w_{0} \partial_{z} n_{0}=0 .
$$

where from the divergence free equation and condition (4.11), $w_{0}$ is given by

$$
w_{0}=\bar{v} \partial_{x} z_{b}-\left(z-z_{b}\right) \partial_{x} \bar{v} .
$$

As commonly we obtain a corrected friction term for the second order approximation. If we look at the equation (4.30), the friction term, $\alpha_{0 B} v_{0}$, depends only on the friction coefficient $\alpha_{0 B}$ while in the system above the friction terms reads as $\alpha_{0 B} \xi \bar{v}$. This new parameter $\xi$ contains the polymer effects into the fluid, represented by $\gamma_{1}, n_{0}$ and $\mathcal{D} e_{0}$. This effect results in a modification of the fluid viscosity, $\mu_{0}+\gamma_{1} n_{0} \mathcal{D} e_{0}$, what is reasonable for very diluted suspensions and it is coherent with the viscoelastic fluid behaviour for small Deborah number. As one can found in [19], the polymers modify several flow characteristics of the solvent, in particular their presence increases the viscosity of the solvent, and the non-Newtonian viscosity increases the friction coefficient. Both properties are recovered by the proposed model.

Nevertheless, it is also known that the fluid becomes elastic, that is, the polymers introduce stress-relaxation characteristics into the fluid. We do not find the effect of the elasticity in the deduced model because in this case it is of order $\varepsilon^{2}$, as we explain in the next section.

On the other hand, we can also observe that since the polymer density $n_{0}$ is just transported by the fluid given by equation (4.38) -, the friction term will affect the dynamics just if the initial mass density holds some inhomogeneity.

Written in dimensional form, system $(2.11)-(2.15)$ represents a formal approximation in $\mathcal{O}\left(\varepsilon^{2}\right)$ of the viscous hydrostatic system and therefore of the Navier-Stokes equations generalizing to FENE type model the paper written in [15].

\section{Intermediate DEBORAH NUMBER REGIME}

The Deborah number (3.2) is a measure of the relevance of the viscoelastic effects compared to the inertial effects. Thus, at small Deborah number $(\mathcal{D} e \ll 1)$ the elasticity of the fluid is irrelevant in that regime and the drag force is comparable to the Newtonian fluid case. At intermediate values of $\mathcal{D} e$ the elasticity of the fluid begins to affect the sedimentation of the particles and an important drag reduction occurs. When higher values of the Deborah number are found, the drag increases again and it exceeds the Newtonian value [16].

However the Deborah number is not the only parameter that influence on the viscoelastic effects. In fact we can introduce the elasticity number $E$ as the ratio between the Deborah number and the Reynolds number, $E=\mathcal{D} e / R e$. As well as we can study the values of Deborah number in order to affect the elasticity of a fluid, we must also take into account the relative order with the Reynolds number.

Many works have been devoted to the study of fluids at small $\mathcal{D} e$ number $[16,18,25]$ and it is put in evidence that the behavior of the flow in this case is equivalent to the case of a Newtonian fluid with a proper viscosity. Nevertheless it is also shown (in theoretical and experimental way) that there is a difference in their behaviour. For example in [19] it is shown that these "slightly viscoelastic fluids" (De $\ll 1)$ decay faster than Newtonian fluids, this means that they have the characteristic of faster damping compared to regular viscous fluids. The damping force is similar to a "friction" force which resists motion via viscous friction. So the viscosity of the fluid acts faster in viscoelastic fluids slowing down quicker the motion than in Newtonian fluids. 
So as the small Deborah number regime is interesting, there is a lot of flows that cannot be categorized in this family. We want in this section to show some ideas about how to tackle the behavior of fluids at intermediate Deborah number. That means that we are not able to solve the Fokker-Planck equation as above and to obtain an explicit value for the stress tensor $\sigma_{P}$. Anyway the idea is to consider the coupled system (2.7) $-(2.10)$ that keeps being true in all cases. Thus, we focus on the derivation of a shallow-water model.

In previous sections we took the assumption of $\mathcal{D} e$ and $\frac{1}{R e}$ to be of order $\varepsilon$, so the fluid we have analyzed count on an elasticity of order $\varepsilon^{2}$, that does not appear in the final system. Here we focus on intermediate Deborah number. We provide the main steps for reader's convenience. In this section we also consider $z_{b}=0$. Asymptotically viscous flows. In this part, we consider the asymptotic regime:

$$
\alpha_{S}=\varepsilon \alpha_{0 S}, \alpha_{B}=\varepsilon \alpha_{0 B}
$$

instead of those done in (4.1). We follow the same process than in Section 4.

First order derivation. We write equations (3.19), (3.22) and (3.25) at principal order, we get:

$$
\frac{1}{R e} \partial_{z}^{2} v_{0}=\mathcal{O}(\varepsilon) ; \quad \frac{1}{R e} \partial_{z} v_{0 \mid z=h}=\mathcal{O}(\varepsilon) ; \quad \frac{1}{R e} \partial_{z} v_{0 \mid z=0}=\mathcal{O}(\varepsilon) ;
$$

so we deduce that the horizontal velocity does not depend on $z$ at first order: $v_{0}=v_{0}(t, x)$. We first look at equation (3.20) to obtain the pressure, we write:

$$
\partial_{z} p=\partial_{z} \sigma_{P}^{22}+\varepsilon \partial_{x} \sigma_{P}^{12}-\frac{1}{\operatorname{Fr}^{2}}+\frac{1}{\operatorname{Re}}\left(\partial_{x} \partial_{z} v+2 \partial_{z}^{2} w\right)+\mathcal{O}\left(\varepsilon^{2}\right)
$$

and we integrate from $z$ to $h$ to get:

$$
\begin{gathered}
p(z)=\sigma_{P}^{22}-\varepsilon \int_{z}^{h} \partial_{x} \sigma_{P}^{12}-\frac{1}{\mathrm{Fr}^{2}}(z-h)+\frac{1}{\operatorname{Re}}\left(\partial_{x} v-\partial_{x}\left(v_{\mid z=h}\right)+2 \partial_{z} w\right) \\
-\left[-p+\sigma_{P}^{22}-\frac{1}{\operatorname{Re}} \partial_{x} h \partial_{z} v+2 \frac{1}{\operatorname{Re}} \partial_{z} w\right]_{\mid z=h}+\mathcal{O}\left(\varepsilon^{2}\right) .
\end{gathered}
$$

From (3.23) we have $\left[-p+\sigma_{P}^{22}-\frac{1}{\operatorname{Re}} \partial_{x} h \partial_{z} v+2 \frac{1}{\operatorname{Re}} \partial_{z} w\right]_{\mid z=h}=\varepsilon \alpha_{0 S} \mathcal{K}+\mathcal{O}\left(\varepsilon^{2}\right)$, so finally we get

$$
p(z)=\sigma_{P}^{22}-\varepsilon \int_{z}^{h} \partial_{x} \sigma_{P}^{12}-\frac{1}{\operatorname{Fr}^{2}}(z-h)+\frac{1}{\operatorname{Re}}\left(\partial_{x} v-\partial_{x}\left(v_{\mid z=h}\right)+2 \partial_{z} w\right)-\varepsilon \alpha_{0 S} \mathcal{K}+\mathcal{O}\left(\varepsilon^{2}\right)
$$

Now we integrate $(3.19)$ in $[0, h]$ and we just consider terms of order $\varepsilon^{0}$ :

$$
\int_{0}^{h}\left(\partial_{t}\left(h v_{0}\right)+v_{0} \partial_{x} v_{0}+w_{0} \partial_{z} v_{0}\right)+\int_{0}^{h} \partial_{x} p_{0}=\int_{0}^{h} \partial_{x} \sigma_{P 0}^{11}+\frac{1}{\operatorname{Re}} \int_{0}^{h}\left(\partial_{z} \partial_{x} w_{0}+2 \partial_{x}^{2} v_{0}\right) .
$$

We use conditions (3.24) and (3.26) to simplify the transport term and the Leibnitz formula to write:

$$
\begin{aligned}
\partial_{t}\left(h v_{0}\right)+\partial_{x}\left(h v_{0}^{2}\right)+\partial_{x} & \int_{0}^{h} p_{0}=\partial_{x} \int_{0}^{h} \sigma_{P 0}^{11}+2 \frac{1}{\operatorname{Re}} \partial_{x} \int_{0}^{h} \partial_{x} v_{0} \\
& +\left[\partial_{x} h p_{0}-\sigma_{P 0}^{11} \partial_{x} h+\frac{1}{\operatorname{Re}} \partial_{x} w_{0}-2 \frac{1}{\operatorname{Re}} \partial_{x} h \partial_{x} v_{0}\right]_{\mid z=h}-\frac{1}{\operatorname{Re}} \partial_{x} w_{0 \mid z=0} .
\end{aligned}
$$

Taking the terms of order $\varepsilon$ in condition (3.22) we find that the term in $z=h$ is of order $\mathcal{O}(\varepsilon)$, if we do so with condition (3.25) we get that $-\frac{1}{\operatorname{Re}} \partial_{x} w_{0 \mid z=0}=-\alpha_{0 B} v_{0}$. We calculate the term of pressure, from (5.1):

$$
p_{0}(z)=\sigma_{P 0}^{22}-\frac{1}{\operatorname{Fr}^{2}}(z-h)+\frac{1}{\operatorname{Re}}\left(\partial_{x} v_{0}-\partial_{x} v_{0}+2 \partial_{z} w_{0}\right)+\mathcal{O}(\varepsilon),
$$


so taking into account that $v_{0}$ does not depend on $z$ and the divergence free condition we get

$$
\partial_{x} \int_{0}^{h} p_{0}=\partial_{x} \int_{0}^{h} \sigma_{P 0}^{22}+\frac{1}{2} \frac{1}{\mathrm{Fr}^{2}} \partial_{x} h^{2}-2 \frac{1}{\operatorname{Re}} \partial_{x}\left(h \partial_{x} v_{0}\right)+\mathcal{O}(\varepsilon) .
$$

We finally write the approximated model at order 1 :

$$
\partial_{t}\left(h v_{0}\right)+\partial_{x}\left(h v_{0}^{2}\right)+\frac{1}{2} \frac{1}{\operatorname{Fr}^{2}} \partial_{x} h^{2}-4 \frac{1}{\operatorname{Re}} \partial_{x}\left(h \partial_{x} v_{0}\right)=\partial_{x} \int_{0}^{h}\left(\sigma_{P 0}^{11}-\sigma_{P 0}^{22}\right)-\alpha_{0 B} v_{0} .
$$

\section{Numerical RESUlts}

In this section we present some numerical simulations corresponding to the case 1: low Deborah number regime. Thus, we consider the system derived previously written in dimensional form given by equations (2.11)-(2.15). Our main objective is to point out the influence of the polymer presence into the flow, so we will compare the solution of the obtained model with the solution of the viscous Saint-Venant system presented in [15].

We consider a simplified model for which we don't take into account the surface tension effect $\left(\alpha_{S}=0\right)$ and we assume that $n_{0}$ does not depend on $z$, so we can write $n_{0}$ as the solution of

$$
\partial_{t} n_{0}+\bar{v} \partial_{x} n_{0}=0
$$

or equivalently

$$
\partial_{t}\left(\tilde{h} n_{0}\right)+\partial_{x}\left(\bar{v} \tilde{h} n_{0}\right)=0 .
$$

Thanks to this assumption, we also find a simplification of the expression of the drag coefficient $\xi$ from (2.13):

$$
\xi=\left(1+\frac{\alpha_{B} \tilde{h}}{3\left(\mu+\tau\left(n_{0}\right)\right)}\right)^{-1}
$$

with $\tau\left(n_{0}\right)=\overline{\gamma_{1}} n_{0} \mathcal{D} e$.

From now on we drop the "tilde" and "bar" notation for the sake of simplicity. Thus, we denote by (SV) the viscous Saint-Venant system and by (S2) the second order approximated system obtained in this work. They read as follows:

$$
\begin{gathered}
(\mathrm{SV})\left\{\begin{array}{l}
\partial_{t} h+\partial_{x}(h v)=0 \\
\partial_{t}(h v)+\partial_{x}\left(h v^{2}+\frac{1}{2} g h^{2}\right)-4 \mu \partial_{x}\left(h \partial_{x} v\right)=-\xi_{S V} \alpha_{B} v-g h \partial_{x} z_{b}
\end{array}\right. \\
(\mathrm{S} 2)\left\{\begin{array}{l}
\partial_{t} h+\partial_{x}(h v)=0 \\
\partial_{t}(h v)+\partial_{x}\left(h v^{2}+\frac{1}{2} g h^{2}\right)-4 \mu \partial_{x}\left(h \partial_{x} v\right)=-\xi \alpha_{B} v-g h \partial_{x} z_{b} \\
\partial_{t}(h n)+\partial_{x}(v h n)=0
\end{array}\right.
\end{gathered}
$$

with

$$
\xi_{S V}=\left(1+\frac{\alpha_{B} h}{3 \mu}\right)^{-1} \text { and } \xi=\left(1+\frac{\alpha_{B} h}{3(\mu+\tau(n))}\right)^{-1}
$$

for $\tau(n)=\gamma_{1} n \mathcal{D}$ e. We are able to write the system (S2) under the classical increased shallow-water equations formulation (see $[13,38]$ ). In our case the polymer density $n$ acts like a passive scalar transported by the fluid through the velocity $v$. If we define the variable $r=h n$ and the discharge $q=h v$, the system above can be written under the following matricial form for conservative variables:

$$
\partial_{t} W+\partial_{x} F(W)=G(x, W)+R(x, W)
$$




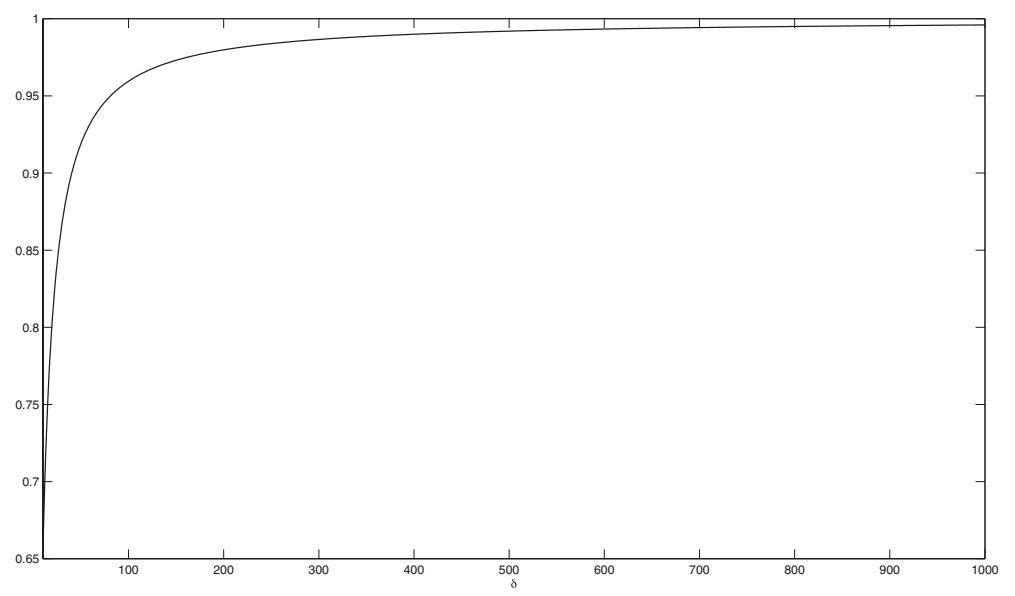

FIGURE 1. $\frac{\gamma_{1}}{\lambda}$ respect to the parameter $\delta$.

where

$$
W=\left(\begin{array}{c}
h \\
q \\
r
\end{array}\right) ; F(W)=\left(\begin{array}{c}
q \\
\frac{q^{2}}{h}+\frac{1}{\frac{q r}{h}} g h^{2}
\end{array}\right) ; G(x, W)=\left(\begin{array}{c}
0 \\
-g h \partial_{x} z_{b} \\
0
\end{array}\right) .
$$

The viscous and friction terms are included in term $R$ :

$$
R(x, W)=\left(\begin{array}{c}
0 \\
4 \mu \partial_{x}\left(h \partial_{x} \frac{q}{h}\right)-\xi \alpha_{B} \frac{q}{h} \\
0
\end{array}\right) .
$$

The polymer properties are collected in the coefficient $\xi$, in particular through the parameters $\gamma_{1}$ and $\mathcal{D} e$ in $\tau(n)$. If we explicit the expression of $\gamma_{1}$ from (4.25) and using Remark 4.5 we get:

$$
\gamma_{1}=\lambda \frac{\delta}{\delta+4}\left(1-\frac{24}{(\delta+6)(\delta+8)}\right)
$$

with $\delta$ related to the maximum elongation of the polymer spring, that generally satisfies $\delta \geq 10,(c f$. [12,34]) and $\lambda$ related to the temperature, the Boltzman constant and the number of polymer molecules per unit volume, we'll take different values to check its influence on the results. The ratio $\frac{\gamma_{1}}{\lambda}$ is called dimensionless elastic viscosity [12], in Figure 1 we show the behaviour of this parameter respect to $\delta$. We can see that there is an important increasing tendency in the range of $\delta \in[10,200]$ approximately, then the value of $\frac{\gamma_{1}}{\lambda}$ tends to the unity as $\delta$ increases. In the results presented below we take the value $\delta=100$ with the aim of obtaining the greatest influence of this parameter and we vary the parameter $\lambda$ that takes the values $10,10^{2}, 10^{3}$. In order to consider the asymptotic regime as in Section 4 , the viscosity is $\mu=\varepsilon \mu_{0}$, the friction coefficient is $\alpha_{B}=\varepsilon \alpha_{0 B}$ and the Deborah number is $\mathcal{D} e=\varepsilon \mathcal{D} e_{0}$. In the sequel we fix $\varepsilon=10^{-3}, \mu_{0}=1, \mathcal{D} e_{0}=1$ and $\alpha_{0 B}$ takes the values 1,10 or 100 , similarly to the study developed in [15].

To solve numerically these systems we have used the two-second order WAF method (see [13,38]). For all test performed below we take Neumann conditions at the boundary.

\subsection{Test 1: Flat bottom and constant polymer density}

We consider a diluted solution of polymers and we solve a "dam" break problem on a wet floor with flat bottom $z_{b}=0$. We consider a domain of length $L=50$ discretized by 200 points, the CFL condition is fixed as 
a)

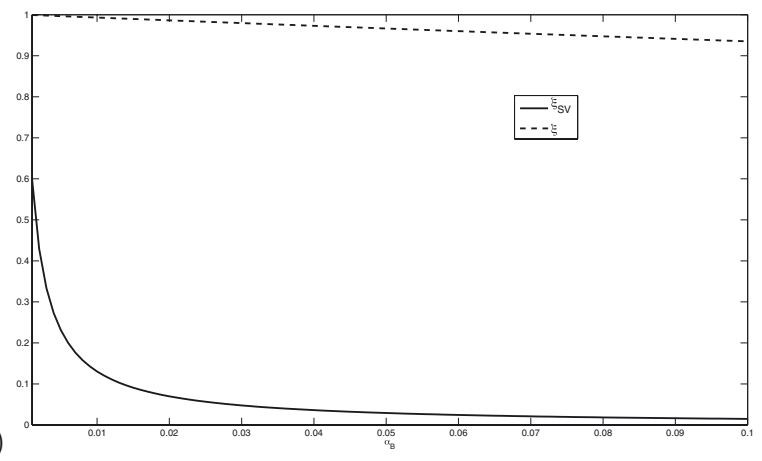

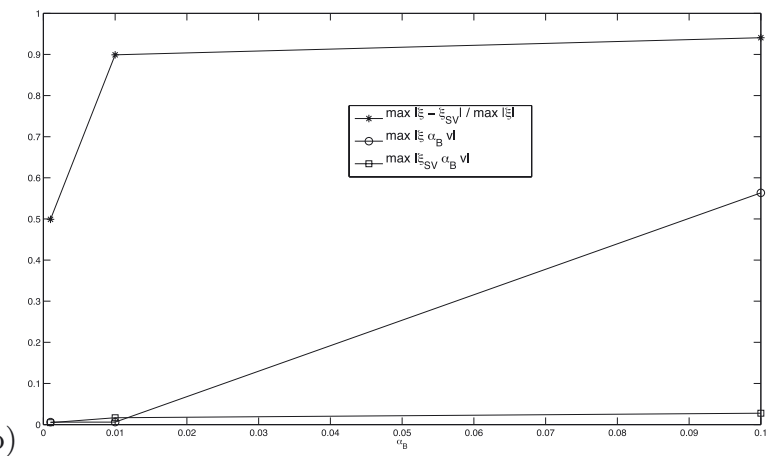

FiguRE 2. a) Values of $\xi_{S V}$ and $\xi$ respect to the friction coefficient $\alpha_{B}$. b) Graph corresponding to data in Table 1.

TABLE 1. Friction values respect to the coefficient $\alpha_{B}$.

\begin{tabular}{|l|c|c|c|}
\hline$\alpha_{B}$ & $10^{-3}$ & $10^{-2}$ & $10^{-1}$ \\
\hline$\frac{\max \left|\xi-\xi_{S V}\right|}{\max |\xi|}$ & 0.4990 & 0.8991 & 0.9407 \\
\hline $\max \left|\xi \alpha_{B} v\right|$ & 0.0060 & 0.0593 & 0.5634 \\
\hline $\max \left|\xi_{S V} \alpha_{B} v\right|$ & 0.0046 & 0.0166 & 0.0276 \\
\hline
\end{tabular}

0.9 and the final time is $t=3$. The initial conditions are given by:

$$
h(t=0)=\left\{\begin{array}{ll}
3 & x<10 \\
0.1 & x \geq 10
\end{array} ; q(t=0)=\left\{\begin{array}{ll}
3.5 & x<10 \\
0 & x \geq 10
\end{array} ; r(t=0)=h(t=0) n_{c}\right.\right.
$$

where the constant polymer density $n=n_{c}$.

This test is devoted to study the influence in the flow behaviour of the friction coefficient $\alpha_{B}$ and the parameters coming from the polymer effects, in particular depending on $n_{c}$ and $\lambda$. First, for fixed $n_{c}=1$ we will study different values for $\lambda$ and $\alpha_{B}$ and secondly we will fix these two values to see the influence of the polymer density $n_{c}$.

If we fix the value of $\lambda$ we obtain different solutions depending on $\alpha_{B}$. Notice that the effect of this parameter is similar for the two systems (SV) and (S2) appearing in the friction term and in the definition of $\xi$ and $\xi_{S V}$. Nevertheless we will see that the system (S2) is more sensitive to $\alpha_{B}$ than the Saint-Venant system due to the influence of the polymer parameter $\gamma_{1}$.

Influence of the friction coefficient. We take $\lambda=10^{3}$ fixed to check what is the effect of the friction coefficient in the behaviour of the flow. First of all we would like to highlight some details into the models in question. As we have mentioned before, the difference between (SV) and (S2) falls on the term $\tau(n)$, only depending on the polymer properties, whos influence appears into the friction term. Thus, the larger is the difference $\left|\xi-\xi_{S V}\right|$, the larger the difference between the solutions. In Figure $2 \mathrm{a}$ we show the values of the coefficients $\xi_{S V}$ and $\xi$ respect to the friction coefficient $\alpha_{B}$. In particular for this graph we have taken $n=1$ and $h=2$ to make the values of $\xi$ and $\xi_{S V}$ only depend on $\alpha_{B}$. We can appreciate that there is an important difference between the two terms but the real difference in the system comes from the friction term $-\xi \alpha_{B} v$. Hence in Table 1 we show the maximum values obtained for the difference between the coefficients $\xi$ and $\xi_{S V}$ and the corresponding friction terms. These results are also shown in Figure 2b. Thus, we can say that the friction coefficient $\alpha_{B}$ has not a great influence on the Saint-Venant system because the friction term keeps small. Nevertheless for the case of the system (S2) we find more significant differences in the friction term, mainly for $\alpha_{B}=10^{-2}$ and $\alpha_{B}=10^{-1}$. 

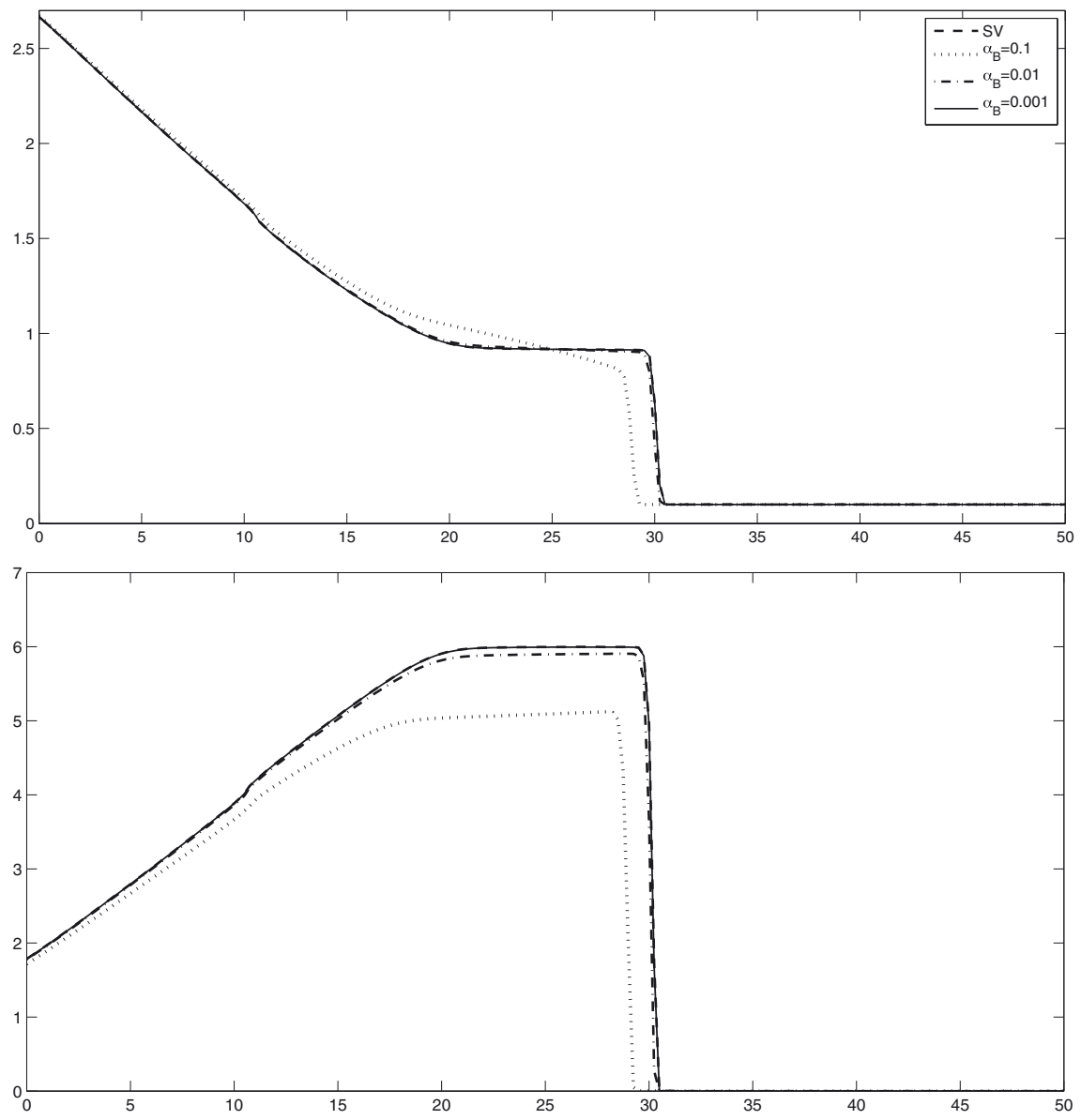

Figure 3. Test 1: height (above) and velocity (below) for different values of $\alpha_{B}$.

In Figure 3 we show the solution of the problem, the height on the top and the velocity below for the different values of $\alpha_{B}$. The (SV) solution for the three different values of $\alpha_{B}$ are superimposed at this scale due to the smallness of the friction term. In fact, it is for this reason that the solution of the system (S2) for $\alpha_{B}=10^{-3}$ is very close to those of $(\mathrm{SV})$. We can appreciate that when $\alpha_{B}$ gets larger, and so the friction term, the solutions become more different.

Influence of the parameter $\lambda$. As a result of the previous study, to check the importance of the friction term, we fix in this part $\alpha_{B}=0.1$ and vary the parameter $\lambda$ to see its influence on the solution between the values 10 , $10^{2}$ and $10^{3}$. Notice that with these values, the new term $\tau(n)$ varies between $0.01,0.1$ and 1 (assuming $n$ at order 1). Following the same idea as above, we first look at the values of the terms regarding the friction effect. We show in Figure 4a the values of $\xi$ and $\xi_{S V}$ respect to $\tau(n)$ for fixed $h=2$ and $n=1$ (so $\xi_{S V}$ is constant). We can see that the difference is almost of the same order than before but now the coefficient $\xi$ has an important increasing for small values of $\tau(n)$. This behaviour will affect to the solution who will get more sensitive to the variation of $\lambda$. Let us check the values of the friction term in this case, they are shown in Table 2 and in Figure $4 \mathrm{~b}$. Now we obtain large values for $\left|\xi-\xi_{S V}\right|$ but also for the friction term, this fact will give us more important differences between the solutions.

Finally in Figure 5, we show the solution of the problem. Since $\alpha_{B}$ is fixed, we have just one solution of the (SV) system. We can really see the influence of the polymers presence into the flow, that gets slower for larger 
a)

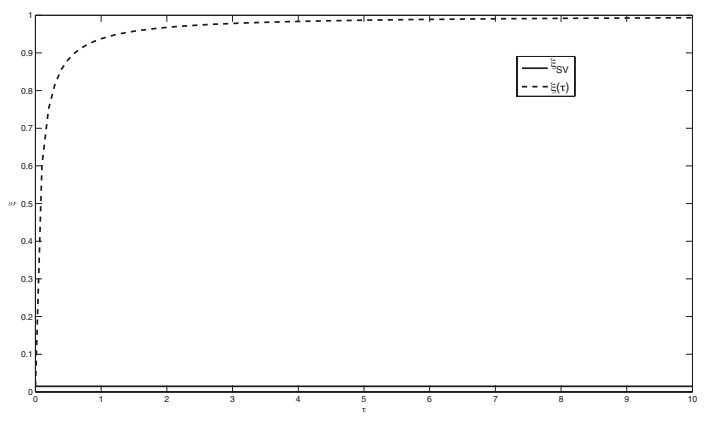

b)

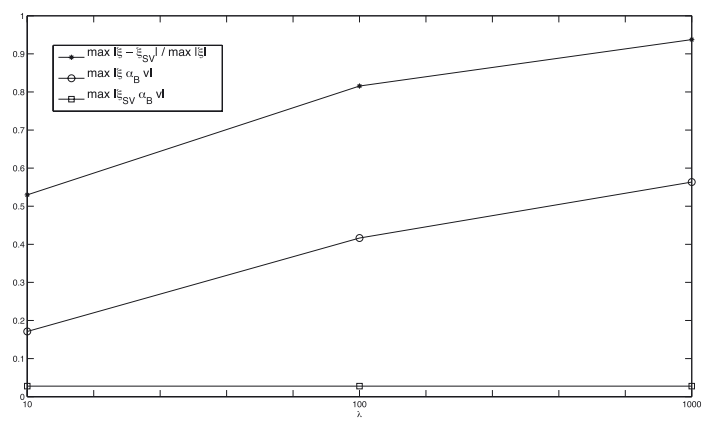

FiguRE 4. a) Values of $\xi_{S V}$ and $\xi$ respect to the parameter $\tau(n)$. b) Graph corresponding to data in Table 2.
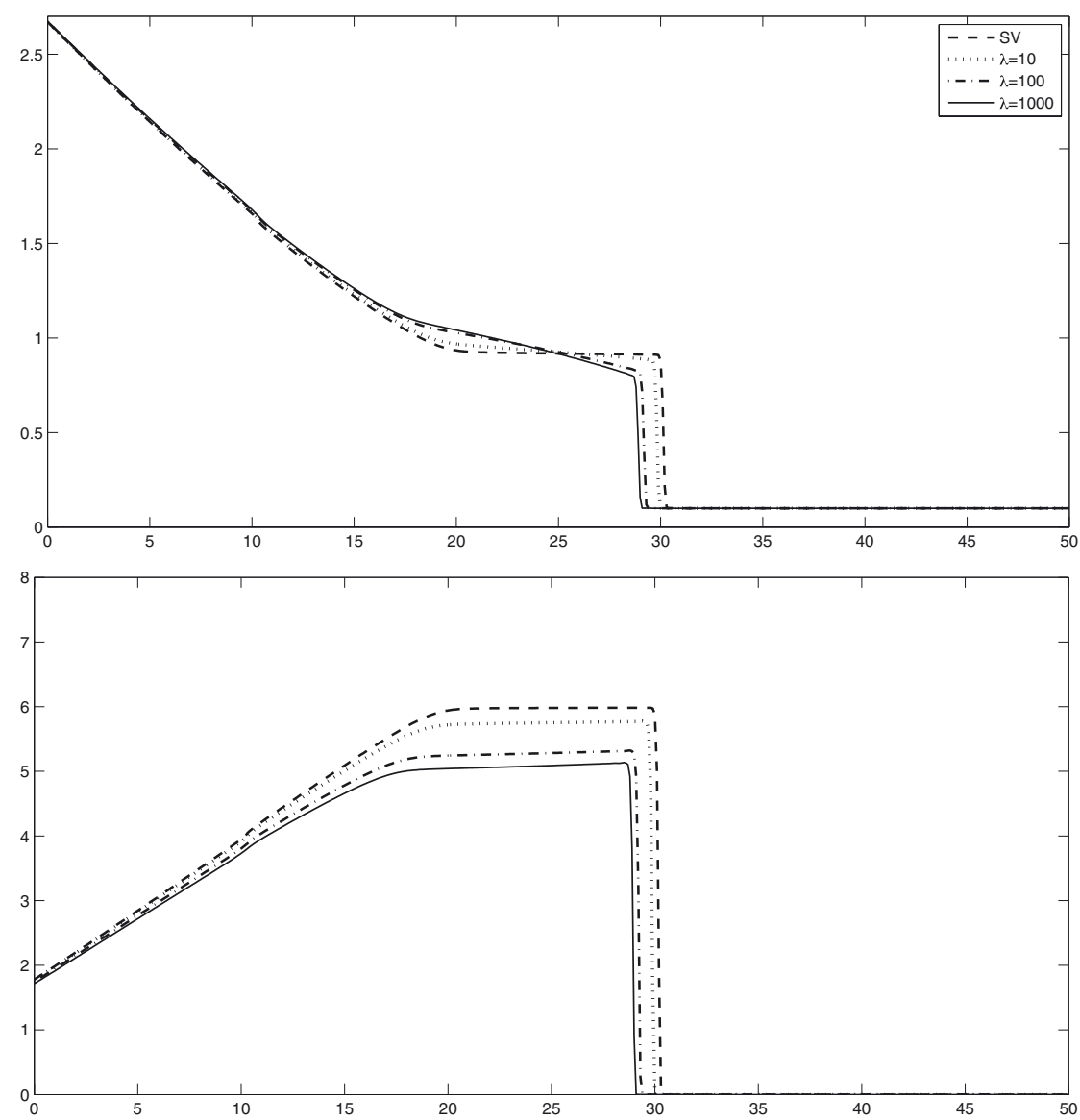

Figure 5. Test 1: height (above) and velocity (below) for different values of $\lambda$.

TABLE 2. Friction values respect to the parameter $\lambda$.

\begin{tabular}{|l|c|c|c|}
\hline$\lambda$ & 10 & $10^{2}$ & $10^{3}$ \\
\hline$\frac{\max \left|\xi-\xi_{S V}\right|}{\max |\xi|}$ & 0.6966 & 0.8436 & 0.9407 \\
\hline $\max \left|\xi \alpha_{B} v\right|$ & 0.1712 & 0.4165 & 0.5634 \\
\hline $\max \left|\xi_{S V} \alpha_{B} v\right|$ & 0.0276 & 0.0276 & 0.0276 \\
\hline
\end{tabular}



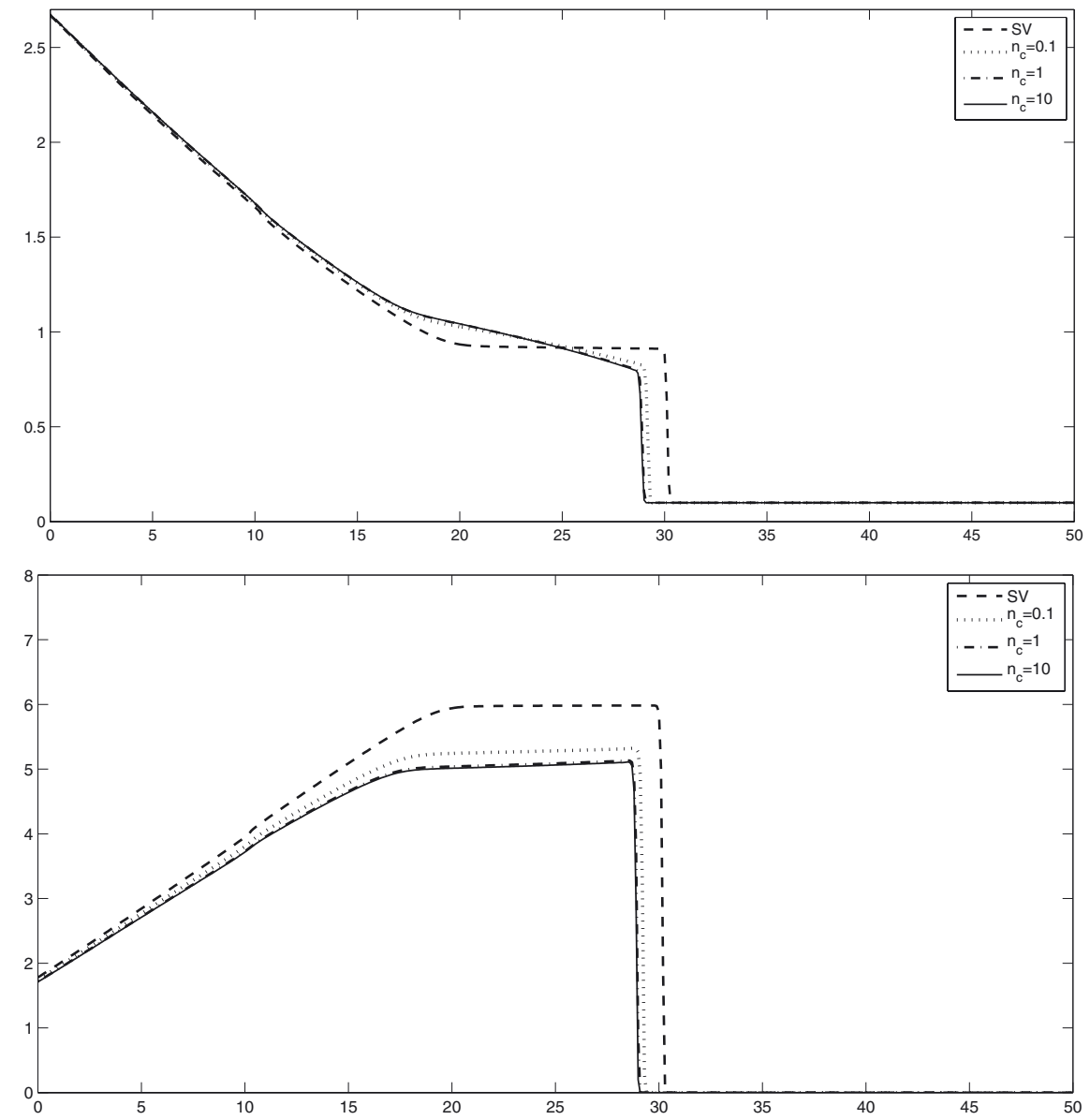

Figure 6. Test 1: height (above) and velocity (below) for different values of $n$.

values of $\lambda$. Thus, for $\lambda=10$ we obtain that the solution of (S2) is close to the Saint-Venant solution, but we can appreciate that the velocity is lower. This difference is more noticeable for higher values of $\lambda$ for which the profile of the front also changes respect to the (SV) solution.

Influence of the polymer density $n_{c}$. In this case we fix the friction coefficient as before to $\alpha_{B}=0.1$ and the parameter $\lambda=10^{3}$. We vary the polymer density $n_{c}$ that takes the values $0.1,1$ and 10 . We obtain similar values than in the case before, because the role of the coefficient $\gamma_{1}$ and $n$ is the same in the function $\tau(n)$. In Figure 6 we show the height and the velocity for all cases. Note that for higher values of the polymer density the velocity decreases and then the fluid moves slowly. As one could hope, for smaller values of $n_{c}$ the solution is closer to those of (SV) system because the term $\tau(n)$ decreases so $\xi$ becomes closer to $\xi_{S V}$. The values of the friction term are shown in Table 3, where we observe that these values matches with the behaviour of the fluid in Figure 6 and the main difference is found for the smaller value of $n_{c}$.

\subsection{Test 2: Non constant bottom and polymer density}

In this second test we consider a non flat bottom and a non constant polymer density in a domain of length $L=50$, the space step is $\Delta x=1 / 20$, the CFL condition is fixed as 0.9 and the final time is $t=4$. The initial 
TABLE 3. Friction values respect to the parameter $n_{c}$.

\begin{tabular}{|l|c|c|c|}
\hline$n_{c}$ & 0.1 & 1 & 10 \\
\hline$\frac{\max \left|\xi-\xi_{S V}\right|}{\max |\xi|}$ & 0.9315 & 0.9407 & 0.9416 \\
\hline $\max \left|\xi \alpha_{B} v\right|$ & 0.4465 & 0.5634 & 0.5788 \\
\hline $\max \left|\xi_{S V} \alpha_{B} v\right|$ & 0.0276 & 0.0276 & 0.0276 \\
\hline
\end{tabular}

TABLE 4. Friction values respect to the parameter $\lambda$.

\begin{tabular}{|l|c|c|c|}
\hline$\lambda$ & 10 & $10^{2}$ & $10^{3}$ \\
\hline$\frac{\max \left|\xi-\xi_{S V}\right|}{\max |\xi|}$ & 0.8417 & 0.9177 & 0.9497 \\
\hline $\max \left|\xi \alpha_{B} v\right|$ & 0.3901 & 0.5975 & 0.6328 \\
\hline $\max \left|\xi_{S V} \alpha_{B} v\right|$ & 0.0455 & 0.0460 & 0.0462 \\
\hline
\end{tabular}

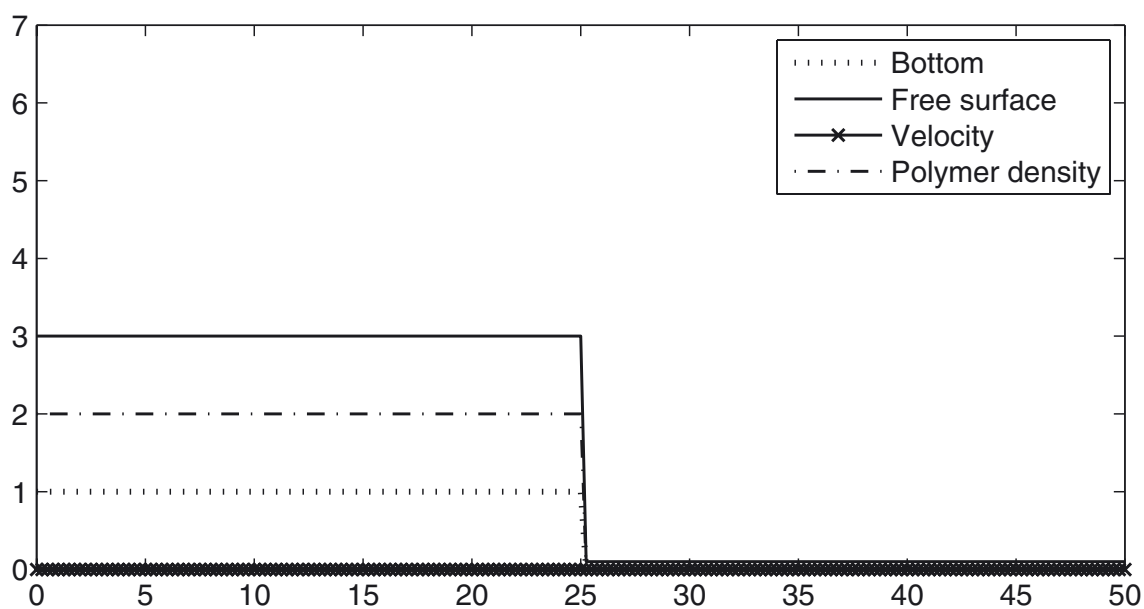

Figure 7. Test 2: Initial conditions.

conditions, represented in Figure 7, are given by:

$$
h(t=0)=\left\{\begin{array}{ll}
2 & x<25 \\
0.1 & x \geq 25
\end{array} ; \quad v(t=0)=0 ; \quad n(t=0)=\left\{\begin{array}{l}
2 x<25 \\
0 x \geq 25
\end{array},\right.\right.
$$

and the bottom is $z_{b}=\left\{\begin{array}{l}1 x<25 \\ 0 x \geq 25\end{array}\right.$.

We consider $\alpha_{B}=0.1$ and the parameter $\lambda$ takes values $10,10^{2}$ or $10^{3}$.

In Table 4 we show the values of the friction terms appearing in the models. We can see that similarly to the Test 1, the friction term for the (SV) system is very small in comparison with those for the (S2) model. We represent in Figure 11 the friction terms $-\xi \alpha_{B} v$ and $-\xi_{S V} \alpha_{B} v$. Since we consider a linear friction law, the profile of the velocity and the friction term are directly connected (see Figs. 9 and 11), as the velocity increases the friction increase and slow the acceleration of the fluid. Actually the polymer effect is just seen in the friction coefficient, thus, the bigger is $\lambda$, the bigger the friction coefficient becomes. As this term has the opposite sign on the conservation equation, it makes the velocity decrease, being lower for higher values of $\lambda$, as we observe in Figure 9. In consequence, there will be a great influence of the polymer presence into the flow, as we show in Figures 8, 9 and 10, where we represent the height, the velocity and the polymer density profiles respectively. 


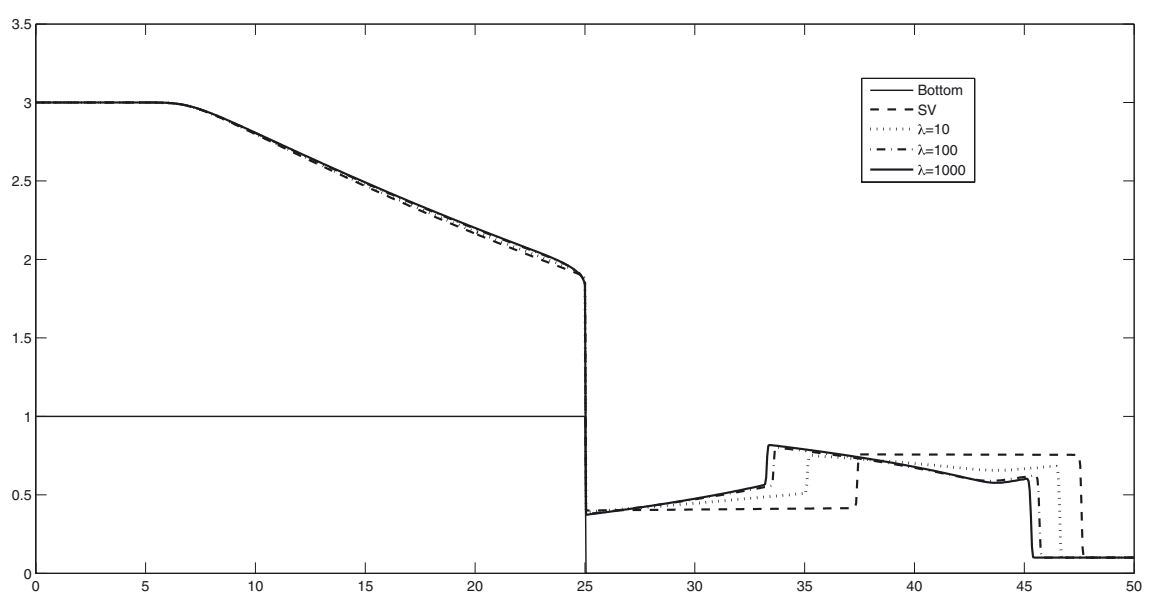

Figure 8. Test 2: Height solution of the systems (SV) and (S2).

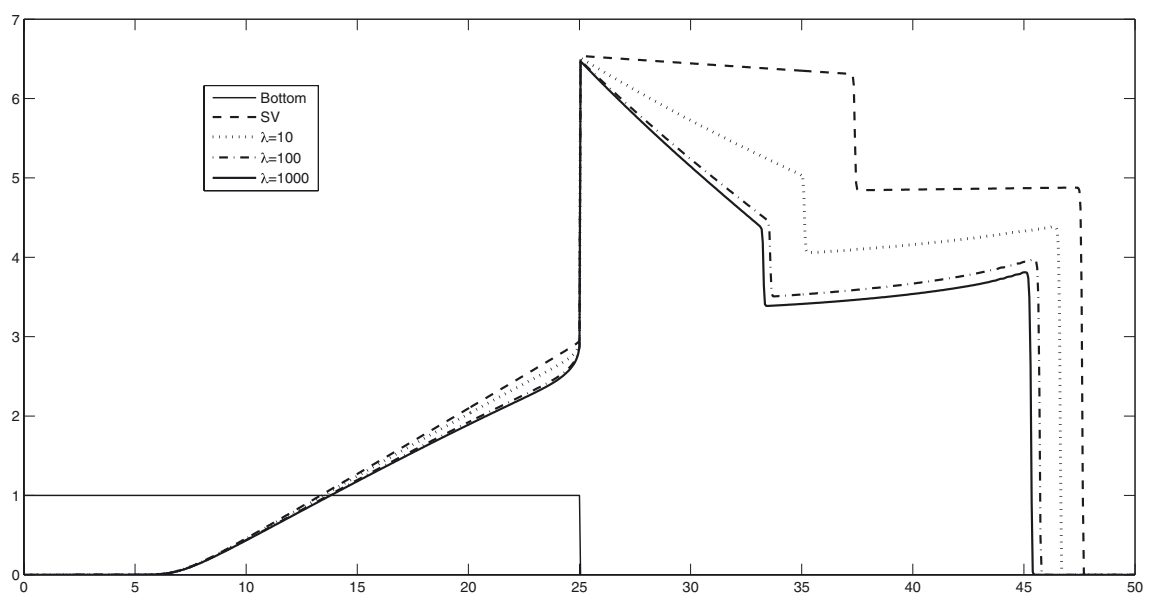

Figure 9. Test 2: Velocity solution of the systems (SV) and (S2).

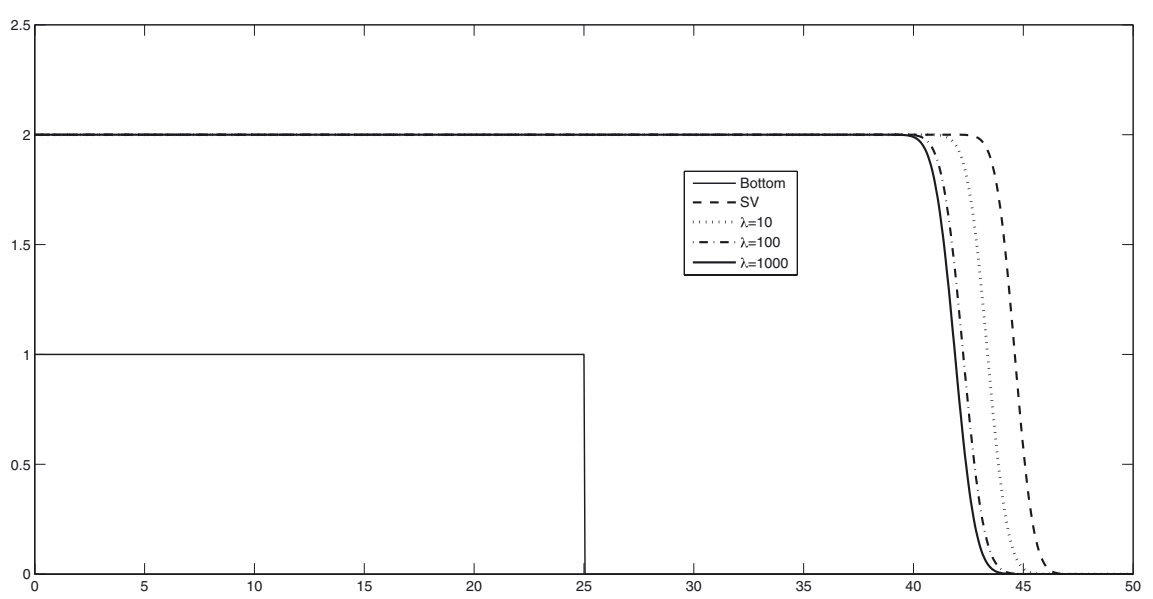

Figure 10. Test 2: Polymer density solution of the systems (SV) and (S2). 


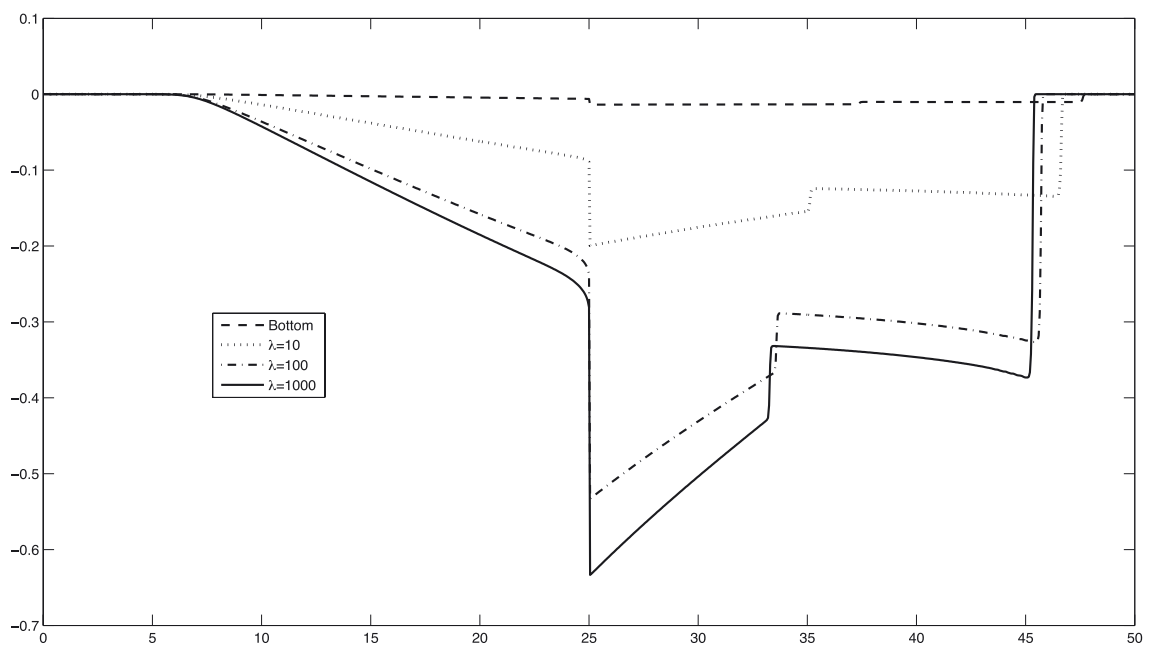

Figure 11. Test 2: Friction term for the different values of $\lambda$ in (S2) and for the (SV) system.

The polymer effect on the velocity affects the behaviour of the height and the transport of the polymer density $n$, mainly after the jump. We can see how for increasing values of $\lambda$ the absolute value of the friction term also increases and this makes the fluid gets slow, who in turn influences on the profile of the height. For high values of $\lambda$ the height and the distance reached by the front decrease.

Acknowledgements. The research of D. Bresch and G. Narbona-Reina to carry on this work was partially supported by the Spanish Government Research project MTM2009-07719 and by the project Maniphyc ANR-08-SYSC-010.

\section{REFERENCES}

[1] C. Ancey, Plasticity and geophysical flows: a review. J. Non-Newtonian Fluid. Mech. 142 (2007) 4-35.

[2] N.J. Balmforth and R.V. Craster, A consistent thin-layer theory for Bingham plastics. J. Non-Newtonian Fluid Mech. 84 (1999) 65-81.

[3] N.J. Balmforth, R.V. Craster and R. Sassi, Shallow viscoplastic flow on an inclined plane. J. Fluid Mech. 420 (2002) 1-29.

[4] J. Banasiak and J.R. Mika, Asymptotic analysis of the Fokker-Planck equations related to Brownian motion. Math. Mod. Meth. Appl. S. 4 (1994) 17-33.

[5] F. Bouchut and S. Boyaval, A new model for shallow elastic fluids. Preprint (2011), ArXiv:1110.0799 [math.NA] .

[6] D. Bresch, E.D. Fernández-Nieto, I.R. Ionescu and P. Vigneaux, Augmented Lagrangian Method and Compressible Visco-plastic Flows: Applications to Shallow Dense Avalanches. Adv. Math. Fluid Mech. (2010) 57-89. Doi: 10.1007/978-3-0346-0152-8_4.

[7] D. Bresch and P. Noble, Mathematical justification of a shallow water model. Methods Appl. Anal. 14 (2007) 87-118.

[8] E.C. Bingham, Fluidity and plasticity. Mc Graw-Hill (1922).

[9] R.B. Bird, C.F. Curtiss, R.C. Armstrong and O. Hassager, Dynamic polymeric liquids, in: Kinetic theory. John Wiley and Sons (1987).

[10] L. Chupin, The FENE model for viscoelastic thin film flows. Meth. Appl. Anal. 16 (2009) 217-262.

[11] M. Boutounet, L. Chupin, P. Noble and J.-P. Vila, Shallow water viscous Bows for arbitrary topography. Commun. Math. Sci. 6 (2008) 29-55.

[12] P. Degond, M. Lemou and M. Picasso, Viscoelastic fluid models derived from kinetic equations for polymers. SIAM J. Appl. Math. 62 (2002) 1501-1019.

[13] E.D. Fernández-Nieto and G. Narbona-Reina, Extension of WAF Type Methods to Non-Homogeneous Shallow-Water Equations with Pollutant. J. Sci. Comput. 36 (2008) 193-217.

[14] E.D. Fernández-Nieto, P. Noble and J.-P. Vila, Shallow Water equations for Non Newtonian fluids, J. Non-Newtonian Fluid Mech. 165 (2010) 712-732.

[15] J.-F. Gerbeau and B. Perthame, Derivation of viscous Saint-Venant system for laminar shallow water, numerical validation. Discrete Contin. Dyn. Syst. Ser. B 1 (2001) 89-102.

[16] M. Guala and A. Stocchino, Large-scale flow structures in particle-wall collision at low Deborah numbers. Eur. J. Mech. B/Fluids 26 (2007) 511-530. 
[17] O.G. Harlen, J.M. Rallison and M.D. Chilcott, High-Deborah-Number flows of dilute polymer solutions. J. Non-Newtorian Fluid Mech. 34 (1990) 319-349.

[18] A. Harnoy, The relation between CMD instability and Deborah number in differential type rheological equations. Rheol. Acta 32 (1993) 483-489.

[19] A. Harnoy, Bearing Design in Machinery Engineering Tribology and Lubrication. CRC Press (2002).

[20] W.H. Herschel and T. Bulkley, Measurement of consistency as applied to rubber-benzene solutions. Am. Soc. Test Proc. 26 (1926) 621-633.

[21] B. Jourdain, T. Lelièvre and C. Le Bris, Numerical analysis of micro-macro simulations of polymeric fluid flows: a simple case. Math. Mod. Meth. Appl. S 12 (2002) 1205-1243.

[22] Y. Kwon, S.J. Kim and S. Kim, Finite element modeling of high Deborah number planar contration flows with rational function interpolation of the Leonov model. Korea-Australia Rheol. J. 15 (2003) 131-150.

[23] E. Lauga, Life at high Deborah number. Europhys. Lett. 86 (2009). Doi: 10.1209/0295-5075/86/64001.

[24] C. Le Bris, Systèmes multi-échelles: Modélisation et simulation. Springer-Verlag, Berlin (2005).

[25] T. Li, E. Vanden-Eijnden, P. Zhang and W. E, Stochastic models of polymeric fluids at small Deborah number. J. NonNewtonian Fluid Mech. 121 (2004) 117-125.

[26] F. Lin, P. Zhang and Z. Zhang, On the Global Existence of Smooth Solution to the 2-D FENE Dumbbell Model. Commun. Math. Phys. 277 (2008) 531-553.

[27] A. Lozinski, R.G. Owens and G. Fang, A Fokker-Planck based numerical method for modelling non-homogeneous flows of dilute polymeric solutions. J. Non-Newtonian Fluid Mech. 122 (2004) 273-286.

[28] F. Marche, Derivation of a new two-dimensional viscous shallow water model with varying topog- raphy, bottom friction and capillary effects, Eur. J. Mechanics-B/Fluids 26 (2007) 49-63.

[29] N. Masmoudi, Well-Posedness for the FENE Dumbbell Model of Polymeric Flows. Commun. Pure Appl. Math. 61 (2008) 1685-1714.

[30] A. Mellet and A. Vasseur, Asymptotic Analysis for a Vlasov-Fokker-Planck/ Compressible Navier-Stokes System of Equations. Commun. Math. Phys. 281 (2008) 573-596.

[31] G. Narbona-Reina and D. Bresch, On a shallow water model for non-newtonian fluids, Numer. Math. Adv. Appl. Springer Berlin, Heidelberg (2010) 693-701.

[32] G. Narbona-Reina, J.D. Zabsonré, E.D. Fernández-Nieto and D. Bresch, Derivation of a bilayer model for Shallow Water equations with viscosity. Numerical validation. CMES 43 (2009) 27-71.

[33] A. Oron, S.H. Davis and S.G. Bankoff, Long-scale evolution of thin liquid films. Rev. Mod. Phys. 69 (1997) 931-980.

[34] H.C. Ottinger, Stochastic Processes in Polymeric Fluids. Springer-Verlag, Berlin (1996).

[35] M. Reiner, The Deborah number, Phys. Today 12 (1964) 62.

[36] S. Shao and E.Y.M. Lo, Incompressible SPH method for simulating Newtonian and non-Newtonian flows with a free surface. Adv. Water Resources 26 (2003) 787-800.

[37] J.A. Tichy and M.F. Modest, A Simple Low Deborah Numer Model for Unsteady Hydrodynamic Lubrication, Including Fluid Inertia. J. Rheology 24 (1980) 829-845.

[38] E.F. Toro, Shock-Capturing Methods for Free-Surface Shallow Flows. John Wiley and Sons, England (2001).

[39] H. Zhang and P. Zhang, Local Existence for the FENE-Dumbbell Model of Polymeric Fluids. Arch. Ration. Mech. Anal. 181 (2006) 373-400. 